\title{
Spatially Resolved CO SLED of the Luminous Merger Remnant NGC 1614 with ALMA
}

\author{
Toshiki Saito $^{1,2}$, Daisuke Iono ${ }^{2,3}$, Cong K. Xu ${ }^{4}$, Kazimierz Sliwa ${ }^{5}$, Junko Ueda ${ }^{6}$, Daniel Espada ${ }^{2,3}$, Hiroyuki Kaneko ${ }^{7}$, \\ Sabine König $^{8}$, Kouichiro Nakanishi ${ }^{2,3}$, Minju Lee ${ }^{1,2}$, Min S. Yun ${ }^{9}$, Susanne Aalto ${ }^{8}$, John E. Hibbard ${ }^{10}$, Takuji Yamashita ${ }^{11}$, \\ Kentaro Motohara $^{12}$, and Ryohei Kawabe ${ }^{1,2,3}$ \\ ${ }^{1}$ Department of Astronomy, The University of Tokyo, 7-3-1 Hongo, Bunkyo-ku, Tokyo 113-0033, Japan; toshiki.saito@nao.ac.jp \\ ${ }^{2}$ National Astronomical Observatory of Japan, 2-21-1 Osawa, Mitaka, Tokyo, 181-8588, Japan \\ ${ }^{3}$ The Graduate University for Advanced Studies (SOKENDAI), 2-21-1 Osawa, Mitaka, Tokyo, 181-0015, Japan \\ ${ }^{4}$ Infrared Processing and Analysis Center (IPAC), California Institute of Technology, 770 South Wilson Avenue, Pasadena, CA 91125, USA \\ ${ }^{5}$ Max-Planck Institute for Astronomy, Königstuhl 17, D-69117 Heidelberg, Germany \\ ${ }^{6}$ Harvard-Smithsonian Center for Astrophysics, 60 Garden Street, Cambridge, MA 02138, USA \\ ${ }^{7}$ Nobeyama Radio Observatory, Minamimaki, Minamisaku, Nagano 384-1305, Japan \\ ${ }^{8}$ Chalmers University of Technology, Department of Earth and Space Sciences, Onsala Space Observatory, SE-43992 Onsala, Sweden \\ ${ }^{9}$ Department of Astronomy, University of Massachusetts, Amherst, MA 01003, USA \\ ${ }^{10}$ National Radio Astronomy Observatory, 520 Edgemont Road, Charlottesville, VA 22903, USA \\ ${ }^{11}$ Institute of Space and Astronautical Science, Japan Aerospace Exploration Agency, 3-1-1 Yoshinodai, Sagamihara, Kanagawa 229-8510, Japan \\ ${ }^{12}$ Institute of Astronomy, The University of Tokyo, 2-21-1 Osawa, Mitaka, Tokyo 181-0015, Japan \\ Received 2016 October 23; revised 2016 December 9; accepted 2016 December 21; published 2017 January 30
}

\begin{abstract}
We present high-resolution (1".0) Atacama Large Millimeter/submillimeter Array (ALMA) observations of $\mathrm{CO}(1-0)$ and $\mathrm{CO}(2-1)$ rotational transitions toward the nearby IR-luminous merger NGC 1614 supplemented with ALMA archival data of CO (3-2) and CO (6-5) transitions. The CO (6-5) emission arises from the starburst ring (central $590 \mathrm{pc}$ in radius), while the lower- $J$ CO lines are distributed over the outer disk ( $\sim 3.3 \mathrm{kpc}$ in radius). Radiative transfer and photon-dominated region (PDR) modeling reveals that the starburst ring has a single warmer gas component with more a intense far-ultraviolet radiation field $\left(n_{\mathrm{H}_{2}} \sim 10^{4.6} \mathrm{~cm}^{-3}, T_{\text {kin }} \sim 42 \mathrm{~K}\right.$, and $\left.G_{0} \sim 10^{2.7}\right)$ relative to the outer disk $\left(n_{\mathrm{H}_{2}} \sim 10^{5.1} \mathrm{~cm}^{-3}, T_{\text {kin }} \sim 22 \mathrm{~K}\right.$, and $\left.G_{0} \sim 10^{0.9}\right)$. A two-phase molecular interstellar medium with a warm and cold $(>70$ and $\sim 19 \mathrm{~K})$ component is also an applicable model for the starburst ring. A possible source for heating the warm gas component is mechanical heating due to stellar feedback rather than PDR. Furthermore, we find evidence for non-circular motions along the north-south optical bar in the lower- $J$ CO images, suggesting a cold gas inflow. We suggest that star formation in the starburst ring is sustained by the bardriven cold gas inflow and that starburst activities radiatively and mechanically power the $\mathrm{CO}$ excitation. The absence of a bright active galactic nucleus can be explained by a scenario where cold gas accumulating on the starburst ring is exhausted as the fuel for star formation or is launched as an outflow before being able to feed to the nucleus.
\end{abstract}

Key words: galaxies: individual (NGC 1614, Arp 186) - galaxies: interactions - galaxies: ISM - radiative transfer - submillimeter: galaxies

\section{Introduction}

Recent single-dish and Herschel spectroscopic observations successfully detected bright high- $J$ CO emission $(J=4-3$ up to 30-29; Panuzzo et al. 2010; van der Werf et al. 2010; Rangwala et al. 2011; Hailey-Dunsheath et al. 2012; Kamenetzky et al. 2012; Papadopoulos et al. 2012, 2014; Rosenberg et al. 2012, 2015; Spinoglio et al. 2012; Meijerink et al. 2013; Pellegrini et al. 2013; Pereira-Santaella et al. 2013, 2014; Rigopoulou et al. 2013; Greve et al. 2014; Kamenetzky et al. 2014, 2016; $\mathrm{Lu}$ et al. 2014; Schirm et al. 2014; Topal et al. 2014; Falstad et al. 2015; Liu et al. 2015; Mashian et al. 2015; Wu et al. 2015; Pearson et al. 2016) from nearby starburst galaxies and (ultra-)luminous infrared galaxies (U/LIRGs), as well as lower-J CO lines (e.g., Yao et al. 2003; Narayanan et al. 2005; Iono et al. 2009; Leech et al. 2010; Mao et al. 2010; Papadopoulos et al. 2012; Wilson et al. 2012; Michiyama et al. 2016). The observational evidence of extreme $\mathrm{CO}$ excitation in $\mathrm{U} / \mathrm{LIRG}$ is explained by a combination of several heating models of the interstellar medium (ISM), which consists of cosmic-ray-dominated regions (CRDRs), photondominated regions (PDRs), X-ray-dominated regions (XDRs), and/or mechanically dominated regions (MDRs), because coarse single-dish beams $(\lesssim 10 \mathrm{kpc})$ are not able to distinguish many molecular conditions (Papadopoulos 2010; van der Werf et al. 2010; Bayet et al. 2011; Aalto 2013; Meijerink et al. 2013). High-resolution imaging of various $\mathrm{CO}$ lines is thus an important way to investigate the multiple phases of the molecular ISM and physical processes involved in obscured central activities of galaxies.

Interferometric studies of the $\mathrm{CO} J=6-5$ transition (hereafter $\mathrm{CO}(6-5))$ in nearby LIRGs showed that the distribution of the $\mathrm{CO}(6-5)$ emission is compact compared to the extended lower-J CO emission (Matsushita et al. 2009; Sliwa et al. 2013, 2014; Xu et al. 2014, 2015; Rangwala et al. 2015; Zhao et al. 2016), suggesting that CO (6-5) probes warmer and denser gas directly related to the nuclear activities. However, a direct comparison among distributions of multiple $\mathrm{CO}$ transitions is not straightforward as we are limited in angular resolution for the low- $J$ lines; sensitivities and $u v$-coverages vary; and the high- $J$ lines require good weather, making observations scarce from the ground. In this paper, we present high-resolution, high-sensitivity, and $u v$-matched ALMA observations of the nearby IR-bright galaxy NGC 1614 in order to understand the distribution and kinematics of multiple 

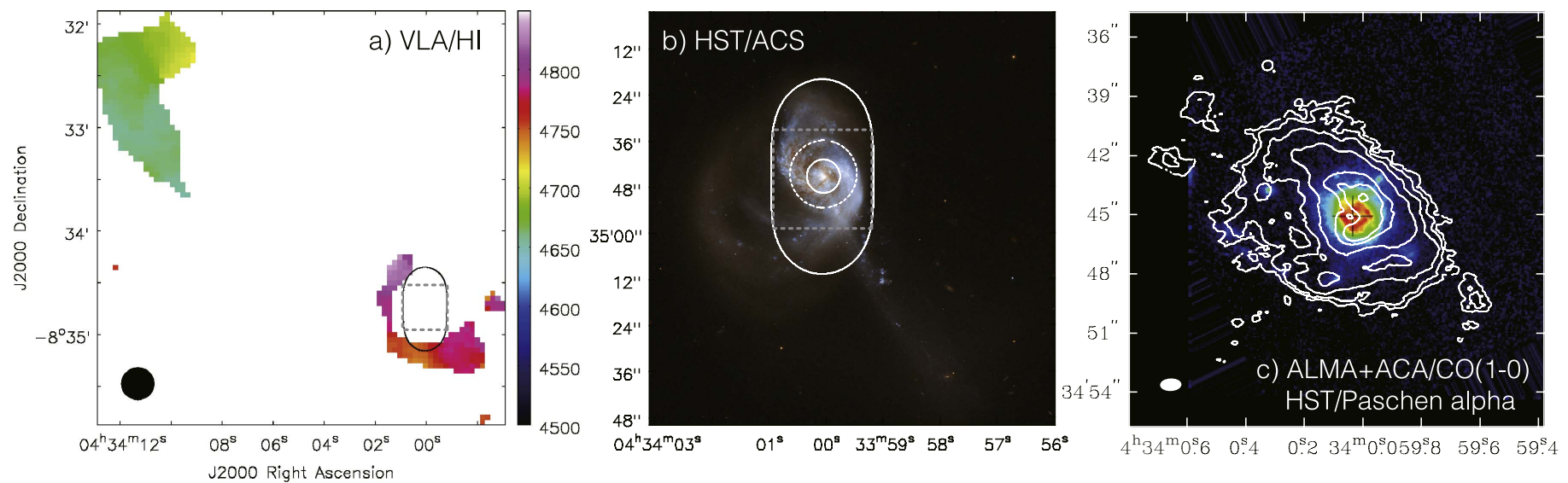

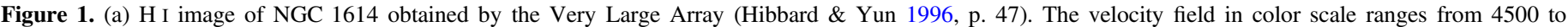

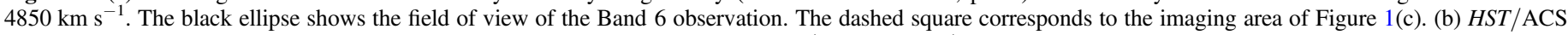

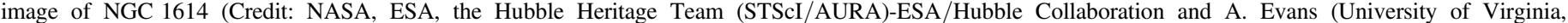

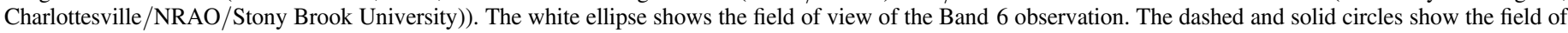

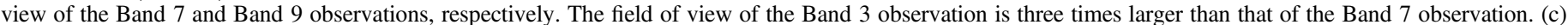

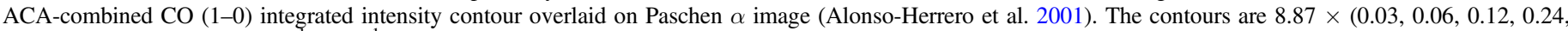

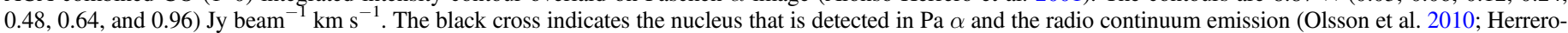
Illana et al. 2014). The imaging area is same as CO images shown in Figure 2.

CO lines and their excitation conditions in the nuclear region. We investigate lower- $J$ CO lines up to $J_{\text {upp }}=6$, which are thought to be mainly excited by star-forming activities.

NGC 1614 is a nearby LIRG $\left(L_{\mathrm{IR}}=10^{11.65} L_{\odot}\right.$; Armus et al. 2009) at a distance of $67.8 \mathrm{Mpc}\left(1^{\prime \prime}=330 \mathrm{pc}\right)$. The total star formation rate (SFR) is $\sim 75 M_{\odot} \mathrm{yr}^{-1}$ based on the measurement of extinction-corrected Paschen $\alpha$ emission (Tateuchi et al. 2015). Merging signatures such as a tidal tail are clearly seen in the HI (Figure 1(a); Hibbard \& Yun 1996, p. 47) and optical images (Figure 1(b); AlonsoHerrero et al. 2001). Numerical simulations performed by Väisänen et al. (2012) suggested that the system is a minor merger with the mass ratio of 1:3-1:5 (i.e., nearly major merger). A strong $\mathrm{HI}$ absorption is detected toward the central region of NGC 1614 surrounded by an arc-like structure in $\mathrm{H} \mathrm{I}$ emission (Hibbard \& Yun 1996, p. 47). Visually, NGC 1614 has been classified as a merger remnant because of no nearby companion (Rothberg \& Joseph 2004) and it is one of the remnants with a rotating molecular gas disk that possibly evolves into a late-type galaxy (Ueda et al. 2014). HCN (4-3) and $\mathrm{HCO}^{+}(4-3)$ observations show that NGC 1614 is consistent with a starburst-dominated galaxy without strong active galactic nuclei (AGNs) signatures (Costagliola et al. 2011; Imanishi \& Nakanishi 2013). This is consistent with the radio, mid-IR, and X-ray observations (Herrero-Illana et al. 2014; Pereira-Santaella et al. 2015). The global CO spectral line energy distribution (SLED) is characterized by a steep decline toward higher- $J$ transitions (Rosenberg et al. 2015). High-resolution ( $\sim 0.25 \sim 83 \mathrm{pc})$ ALMA Band 9 observations revealed that the $\mathrm{CO}(6-5)$ distribution coincides with a starburst ring $(r<350 \mathrm{pc})$ detected in $\mathrm{Pa} \alpha$ (Figure 1(c); Alonso-Herrero et al. 2001) and $8.4 \mathrm{GHz}$ radio continuum emission (Xu et al. 2015), while the CO (2-1) and CO (3-2) emission are more extended (Wilson et al. 2008; König et al. 2013; Sliwa et al. 2014). Recent high-sensitivity CO (2-1) observations revealed the presence of a high-velocity wing, which might be associated with a nuclear molecular outflow (García-Burillo et al. 2015), whereas deep CO (1-0) imaging found that extended molecular gas components associated with the southern tidal tail and a dust lane (König et al. 2016). Continuum emission from 4.81 to $691 \mathrm{GHz}$ is confined within the central 1 .'7 radius $(=560 \mathrm{pc})$ and the radioto-FIR spectral energy distribution (SED) can be explained by star-forming activities (Saito et al. 2016a, hereafter S16a).

This paper is organized as follows. The observations and data reduction are summarized in Section 2. All CO maps, position-velocity diagrams (PVDs), and line intensity ratios are presented in Section 3. In Section 4, we describe modeling procedures of the spatially resolved CO SLED using three different models. We provide a discussion of our modeling results in Section 5, including heating source, CO-to- $\mathrm{H}_{2}$ conversion factor, a possible two-phase ISM model and its heating source, cold molecular gas kinematics (inflow and outflow), and overall picture of molecular gas in NGC 1614. Finally, we summarize our main findings in Section 6. We have adopted $\mathrm{H}_{0}=70 \mathrm{~km} \mathrm{~s}^{-1} \mathrm{Mpc}^{-1}, \Omega_{m}=0.3, \Omega_{\Lambda}=0.7$ throughout this paper.

\section{ALMA Observations and Data Reduction}

\subsection{Band 3 and Band 6 Data: $12 m$ Array}

The CO (1-0) and CO (2-1) observations toward NGC 1614 were carried out for our ALMA Cycle 2 program (ID: 2013.1.01172.S) using thirty-five $12 \mathrm{~m}$ antennas. The Band 3 and Band 6 receivers were tuned to the $C O(1-0)$ and CO (2-1) emission lines in the upper sideband, respectively. The Band 3 data (single pointing), with a projected baseline length $\left(L_{\text {baseline }}\right)$ of 28-1060 m (C34-6 configuration), were obtained on 2014 August 30 (on-source time of $T_{\text {integ }}=16.9$ minutes), and the Band 6 data (3-point mosaic) with $L_{\text {baseline }}=15-349 \mathrm{~m} \quad(\mathrm{C} 34-2 / 1$ configuration $)$ were obtained on 2014 December $8 \quad\left(T_{\text {integ }}=7.3\right.$ minutes per pointing). The correlator was configured to have four spectral windows (SPW), two of which were set to each sideband, each of the SPWs with a $1.875 \mathrm{GHz}$ bandwidth and $1.129 \mathrm{MHz}$ resolution $\left(\sim 3.0\right.$ and $1.4 \mathrm{~km} \mathrm{~s}^{-1}$ for Band 3 and Band 6, respectively). Both bands used a strong quasar J0423-0120 for bandpass and phase calibration. J0423-0120 (=J0423-013) was also the flux calibrator in Band 3, while Uranus was that in Band 6. 
Table 1

Log of ALMA Observations

\begin{tabular}{|c|c|c|c|c|c|c|c|c|c|c|}
\hline \multirow{2}{*}{ Band } & \multirow{2}{*}{ UT Date } & \multicolumn{4}{|c|}{ Configuration } & \multirow{2}{*}{$\begin{array}{l}T_{\mathrm{sys}} \\
\text { (K) } \\
(7)\end{array}$} & \multicolumn{3}{|c|}{ Calibrator } & \multirow{2}{*}{$\begin{array}{c}T_{\text {integ }} \\
\text { (minutes) } \\
\text { (11) }\end{array}$} \\
\hline & & $\begin{array}{l}\text { Array } \\
\text { (3) }\end{array}$ & $\begin{array}{c}\text { FoV } \\
(") \\
(4)\end{array}$ & $\begin{array}{l}N_{\text {ant }} \\
(5)\end{array}$ & $\begin{array}{c}L_{\text {baseline }} \\
\text { (m) } \\
(6)\end{array}$ & & $\begin{array}{l}\text { Flux } \\
(8)\end{array}$ & $\begin{array}{l}\text { Bandpass } \\
\text { (9) }\end{array}$ & $\begin{array}{l}\text { Gain } \\
\text { (10) }\end{array}$ & \\
\hline$\overline{\mathrm{B} 3}$ & 2014 Jun 30 & ACA $7 \mathrm{~m}$ & 95.6 & 10 & $7.3-48$ & $42-170$ & J0501-0159 & J0423-013 & J0427-0700 & 49.1 \\
\hline B3 & 2014 Dec 04 & ALMA $12 \mathrm{~m}$ & 55.7 & 31 & $15-290$ & $31-120$ & Uranus & J0423-0120 & J0423-0120 & 13.1 \\
\hline $\mathrm{B}^{\mathrm{a}}$ & 2014 Aug 30 & ALMA $12 \mathrm{~m}$ & 55.7 & 35 & $28-1060$ & $50-130$ & J0423-013 & J0423-0120 & J0423-0120 & 16.9 \\
\hline$B 6^{\mathrm{a}}$ & 2014 Aug 14 & ALMA $12 \mathrm{~m}$ & 27.8 & 35 & $15-349$ & $70-160$ & Uranus & J0423-0120 & J0423-0120 & $7.3 \times 3$ \\
\hline B7 & 2012 Jul 31/Aug 14 & ALMA $12 \mathrm{~m}$ & 18.6 & 27 & $18-431$ & $100-210$ & Callisto & J0522-364 & J0423-013 & 88.8 \\
\hline B9 & 2012 Aug $13 / 28$ & ALMA $12 \mathrm{~m}$ & 9.3 & 23 & $20-394$ & $500-1100$ & Ceres & J0423-013 & J0423-013 & 49.4 \\
\hline
\end{tabular}

Note. Column 2: Observed date. Column 3: Dish size. Column 4: FWHM of the primary beam at the frequency of the target CO line. Column 5: Number of available antennas. Column 6: Projected length of assigned baseline for NGC 1614. Column 7: DSB system temperature toward NGC 1614. Columns 8-10: Observed flux calibrator, bandpass calibrator, and phase calibrator. Column 11: Total integration time on NGC 1614. The Band 6 observation has three pointing fields.

${ }^{a}$ Data observed for our ALMA Cycle 2 program (ID: 2013.1.01172.S).

Table 2

CO Line and Imaging Properties

\begin{tabular}{|c|c|c|c|c|c|c|c|c|c|c|}
\hline $\begin{array}{l}\text { CO Line } \\
\text { (1) }\end{array}$ & $\begin{array}{c}\nu_{\text {rest }} \\
(\mathrm{GHz}) \\
(2)\end{array}$ & $\begin{array}{c}E_{\mathrm{u}} / k \\
(\mathrm{~K}) \\
(3)\end{array}$ & $\begin{array}{l}\text { Band } \\
\text { (4) }\end{array}$ & $\begin{array}{c}\text { MRS } \\
\left({ }^{\prime \prime}\right) \\
(5)\end{array}$ & $\begin{array}{l}u v \text {-weight } \\
\text { (6) }\end{array}$ & $\begin{array}{c}\text { Beam Size } \\
(") \\
(7)\end{array}$ & $\begin{array}{c}\mathrm{rms} \\
\left(\mathrm{mJy} \mathrm{b}^{-1}\right) \\
(8)\end{array}$ & $\begin{array}{c}S_{\mathrm{CO}} \Delta v \\
\left(\mathrm{Jy} \mathrm{km} \mathrm{s}{ }^{-1}\right) \\
(9)\end{array}$ & $\begin{array}{c}\text { Recovered Flux } \\
(\%) \\
(10)\end{array}$ & $\begin{array}{c}\text { Reference } \\
\text { (11) }\end{array}$ \\
\hline$J=1-0(\mathrm{ACA}+12 \mathrm{~m})$ & 115.271 & 5.53 & B3 & 17.6 & briggs & $1.0 \times 0.6$ & 1.4 & $295.3 \pm 1.8$ & $113 \pm 26$ & $1, \mathrm{a}$ \\
\hline$J=1-0(12 \mathrm{~m})$ & 115.271 & 5.53 & B3 & 4.6 & briggs & $1.0 \times 1.0$ & 1.6 & $69.3 \pm 1.1$ & $27 \pm 5$ & This work, a \\
\hline$J=2-1(12 \mathrm{~m})$ & 230.542 & 16.60 & B6 & 4.6 & uniform & $1.0 \times 1.0$ & 3.5 & $325.4 \pm 2.7$ & $40 \pm 9$ & This work, b \\
\hline$J=3-2(12 \mathrm{~m})$ & 345.796 & 33.19 & B7 & 4.6 & briggs & $1.0 \times 1.0$ & 3.0 & $866.8 \pm 2.2$ & $59 \pm 6$ & $2, \mathrm{c}$ \\
\hline$J=6-5(12 \mathrm{~m})$ & 691.473 & 116.16 & B9 & 4.6 & briggs & $1.0 \times 1.0$ & 8.0 & $826.9 \pm 3.2$ & $58 \pm 10$ & $2,3, \mathrm{~d}$ \\
\hline
\end{tabular}

Note. Column 1: Observed CO transition. Column 2: Rest frequency of the transition. Column 3: Upper state energy of the transition. Column 5: Maximum recoverable scale (MRS) of the visibility data; see the text. Column 6: Visibility ( $u v$-plane) weighting for imaging. "briggs" means Briggs weighting with robust $=0.5$. Column 7: Convolved beam size. All of the images in this work, except for the ACA-combined CO (1-0) image, are convolved into the same resolution of $1{ }^{\prime \prime} 0$, which is larger than the synthesized beam size. Column 8: Noise rms for data with the velocity resolution of $30 \mathrm{~km} \mathrm{~s}^{-1}$. Column 9: Integrated intensity inside the 3\% contour of the peak value. We only consider the statistical error in this column. Column 10: The ALMA flux divided by the single-dish flux. We consider the statistical and the systematic error of the flux calibration. Column 11: Reference of ALMA data (1. König et al. (2016), 2. Sliwa et al. (2014), 3. Xu et al. (2015)) and single-dish data (a. NRAO 12 m; Sanders et al. (1991), b. SEST 15 m; Albrecht et al. (2007), c. JCMT 15 m; Wilson et al. (2008), d. Herschel/ SPIRE; Xu et al. (2015)).

\subsection{Ancillary Data}

\subsubsection{Band 7 and Band 9 Data: $12 m$ Array}

NGC 1614 was observed during Cycle 0 using ALMA in Bands 7 and 9 (ID: 2011.0.00182.S and 2011.0.00768.S), and the data were originally published in Sliwa et al. (2014) and Xu et al. (2015). We obtained the calibrated archival visibility data of the $\mathrm{CO}(3-2)$ and the $\mathrm{CO}(6-5)$ from the ALMA archive. Detailed information of the Cycle 0 and 2 data are shown in Table 1 (see also S16a).

\subsubsection{Band 3 Data: Atacama Compact Array and $12 m$ Array}

The CO (1-0) observations toward NGC 1614 were also carried out during Cycle 2 (ID: 2013.1.00991.S; König et al. 2016) using both ten $7 \mathrm{~m}$ antennas of ACA (Iguchi et al. 2009) and thirty-one $12 \mathrm{~m}$ antennas of the $12 \mathrm{~m}$ array. These ancillary data are used to increase the sensitivity and recover extended emission that we ignore due to the $u v$-clipping (Section 2.1). Thus, we use those data to study the global gas kinematics of NGC 1614. We retrieved those data from the ALMA archive, and used one SPW which was tuned to the
CO (1-0) line. Detailed description of this project is found in König et al. (2016) and we list some parameters in Table 2.

\subsection{Reduction Procedure}

\subsubsection{2 m Array Data}

We performed calibration and imaging using CASA (version 3.4, 4.1.0, and 4.2.2 for Band 7, 9, and 3/6, respectively; McMullin et al. 2007). The detailed procedure for data processing is summarized in S16a. Before imaging, we flagged the $u v$ range below $45 \mathrm{k} \lambda$, so that the maximum recoverable scale (MRS; Lundgren 2013) of each ALMA observation is consistent. The minimum $u v$ distance is determined by the configuration of the CO (6-5) observation. Since the truncated $u v$ range at $45 \mathrm{k} \lambda$ corresponds to the MRS of $\sim 4$ !" $6(\simeq 1.5 \mathrm{kpc})$, we ignore the missing flux effect on structures smaller than 4."6 throughout this work. All of the images are convolved to the same resolution $(1$ !" $0 \times 1$ "' 0$)$. We made all maps in this work without the primary beam correction, although flux measurements were done after that. The systematic errors on the absolute flux calibration using a solar system object are estimated to be $5 \%, 10 \%, 10 \%$, and $15 \%$ for both sidebands in Band 3, Band 6 , Band 7, and Band 9, respectively (Lundgren 2013). The Band 3 


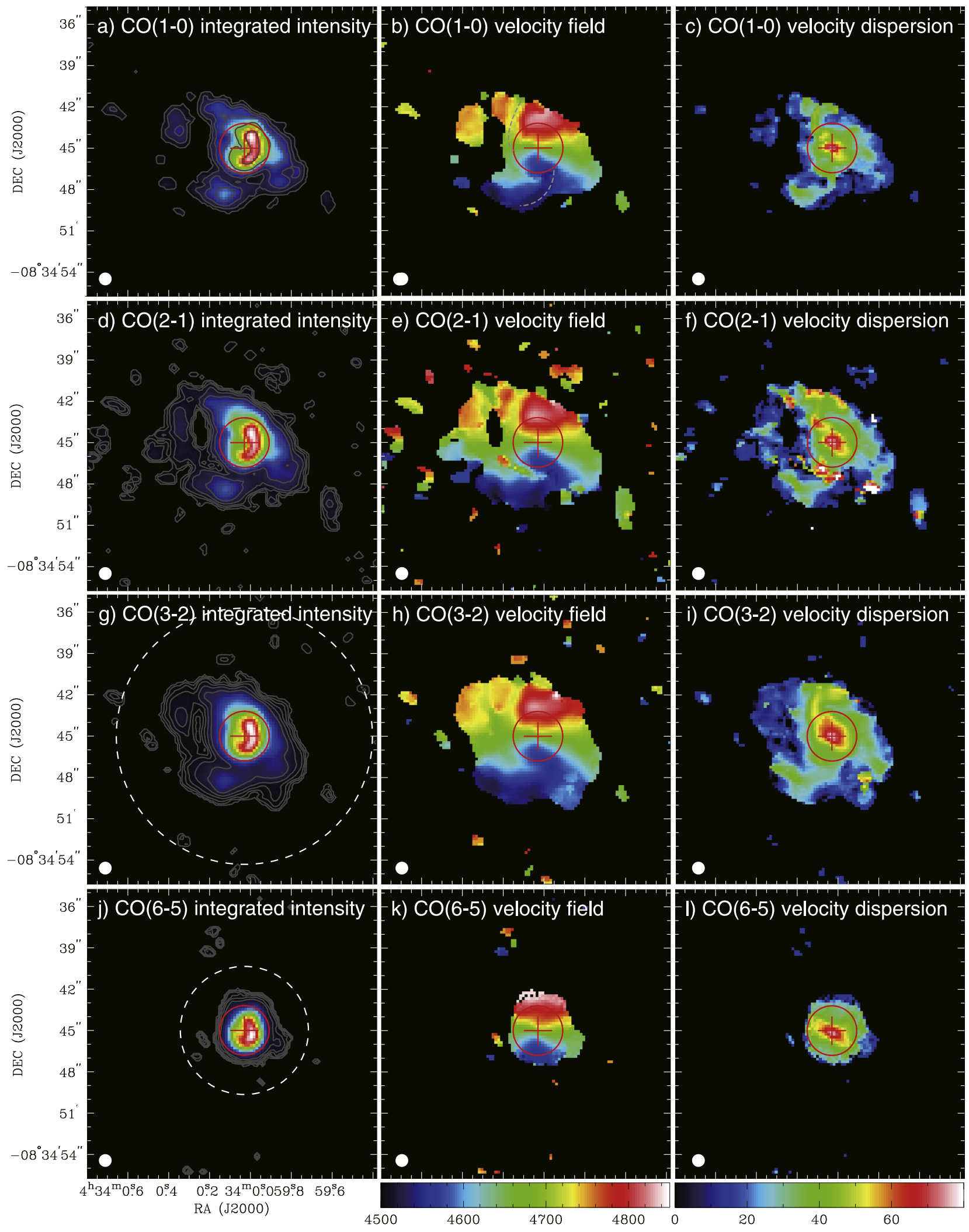

Figure 2. (a) $u v$-clipped CO (1-0) integrated intensity image of NGC 1614. The contours are $0.36,0.72,1.44,2.88,5.76$, and $11.52 \mathrm{Jy} \mathrm{beam}^{-1} \mathrm{~km} \mathrm{~s}^{-1}$. The red cross indicates the nucleus that is detected in $\mathrm{Pa} \alpha$ and radio continuum emission (Olsson et al. 2010; Herrero-Illana et al. 2014). The red circle indicates the approximate outer edge of the starburst ring (König et al. 2013; Herrero-Illana et al. 2014; Xu et al. 2015; S16a). (b) $u v$-clipped CO (1-0) velocity field image. The velocity field in color scale ranges from 4500 to $4850 \mathrm{~km} \mathrm{~s}^{-1}$. The dashed gray lines show the positions of non-circular motion. (c) $u v$-clipped CO (1-0) velocity dispersion image. The velocity dispersion in color scale ranges from 0 to $80 \mathrm{~km} \mathrm{~s}^{-1}$. (d) Same as (a), except for CO (2-1). The contours are 1.0, 2.0, 4.0, 8.0, 16.0, and 32.0 Jy beam $^{-1} \mathrm{~km} \mathrm{~s}^{-1}$. (e/f) Same as (b/c), respectively, except for CO (2-1). (g) Same as (a), except for CO (3-2). The contours are 0.72, 1.44, 2.88, 5.76, 11.52, and 23.04 Jy beam ${ }^{-1} \mathrm{~km} \mathrm{~s}^{-1}$. (h/i) Same as (b/c), respectively, except for CO (3-2). (j) Same as (a), except for CO (6-5). The contours are 2.4, 4.8, 9.6, 19.2, 38.4, and $76.8 \mathrm{Jy} \mathrm{beam}^{-1} \mathrm{~km} \mathrm{~s}^{-1}$. (k/l) Same as (b/c), respectively, except for CO (6-5). The fields of view of the CO (3-2) and CO (6-5) data are shown as white dashed circles. 

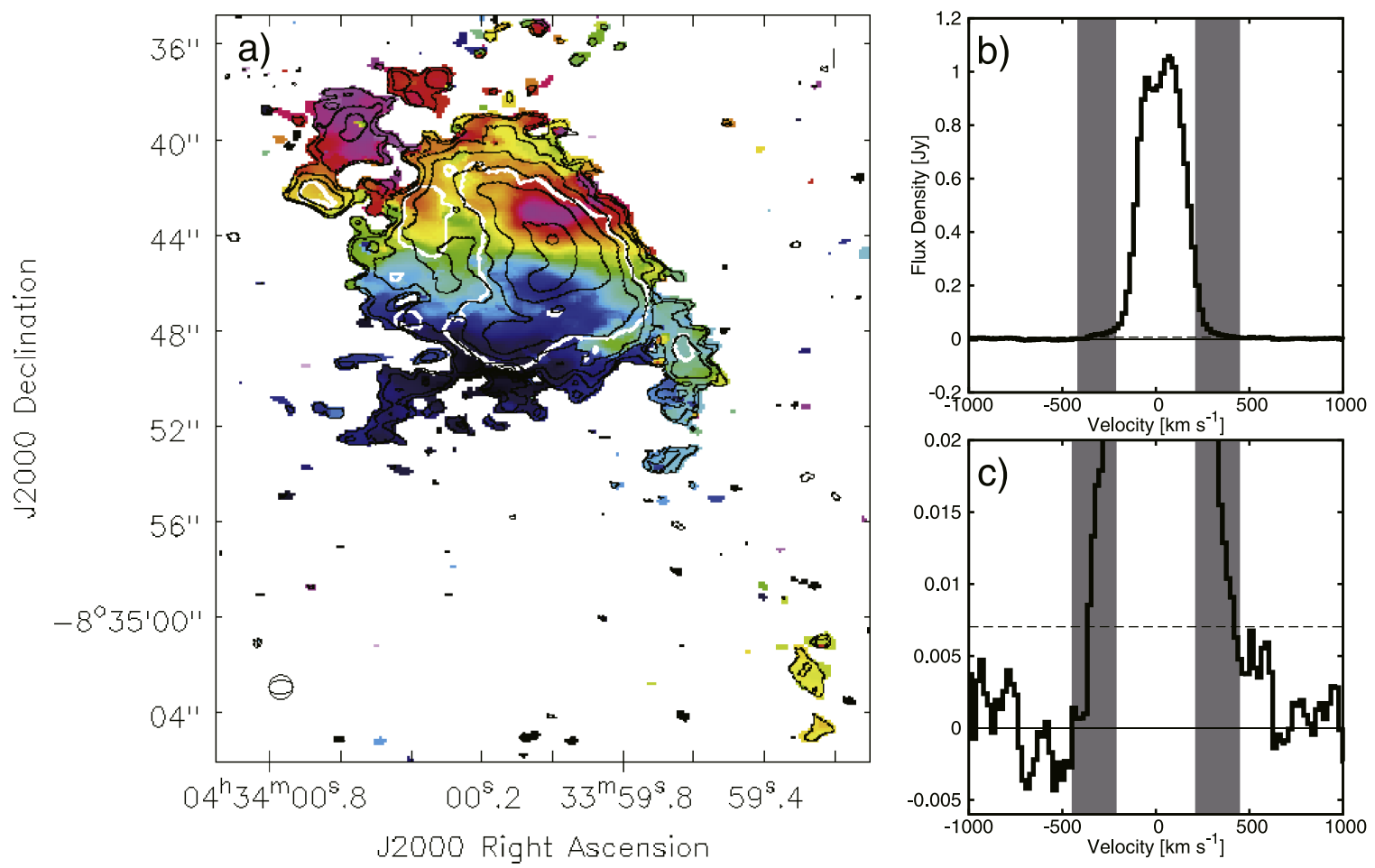

Figure 3. (a) ACA $+12 \mathrm{~m}$ combined $\mathrm{CO}(1-0)$ integrated intensity (black contour) and velocity field (color) images of NGC 1614 . The contours are $8.8 \times(0.02,0.04$ $0.08,0.16,0.32,0.64$, and 0.96$) \mathrm{Jy}_{\text {beam }}^{-1} \mathrm{~km} \mathrm{~s}^{-1}$. The velocity field in color scale ranges from 4500 to $4850 \mathrm{~km} \mathrm{~s}^{-1}$. The white contour shows the outline of the $u v$ clipped $12 \mathrm{~m}$ only CO (1-0) image at the level of $0.36 \mathrm{Jy} \mathrm{beam}^{-1} \mathrm{~km} \mathrm{~s}^{-1}$ (Figure 2(a)). (b) CO (1-0) spectrum toward the central 4 !' 6 aperture. (c) Zoomed-in CO (1-0) spectrum of Figure 3(b). The shaded area shows the CO line wings suggested by García-Burillo et al. (2015).

observation has a quasar as the flux calibrator, so we estimate the absolute systematic error on the flux calibration for our Band 3 data by using the ALMA Calibrator Source Catalog. ${ }^{13}$ The flux uncertainty of J0423-013 on 2014 August 30 is $1.9 \%-3.7 \%$ at $103.5 \mathrm{GHz}$. Therefore, we adopt the flux uncertainty of $5 \%$ to be conservative. Since the recovered flux of each CO transition compared to single-dish observations (Sanders et al. 1991; Albrecht et al. 2007; Wilson et al. 2008; Xu et al. 2015) is very low (27\%-59\%), NGC 1614 has large amounts of ambient molecular gas structures larger than $1.5 \mathrm{kpc}$ even in the $\mathrm{CO}(6-5)$. The detailed data properties and line information are listed in Table 2.

\subsubsection{ACA Data and Combine with $12 \mathrm{~m}$ Array Data}

We used CASA (version 4.2.1 and 4.2.2) to process the data. In order to combine the CO (1-0) data shown in Section 2.2.2 with the data described in Section 2.1, we used statwt ${ }^{14}$ task to recalculate visibility weights for all the data. The resultant $L_{\text {baseline }}$ is $7.3-1060 \mathrm{~m}$. This corresponds to the MRS of 17 !" 6 $(\sim 5.8 \mathrm{kpc})$. We constructed a data cube with the velocity resolution of $20 \mathrm{~km} \mathrm{~s}^{-1}$. The flux calibrator for the ACA observation is a quasar J0501-0159. Using the ALMA Calibrator Source Catalog ${ }^{13}$, the flux uncertainty of J05010159 on 2015 June 30 is estimated to be $\sim 12 \%$ at $97.4 \mathrm{GHz}$. Therefore, we adopt the flux uncertainty of $12 \%$ when we use the ACA-combined CO (1-0) data. The achieved rms noise level is $1.4 \mathrm{mJy} \mathrm{beam}^{-1}$ with the synthesized beam size of 1 !. $02 \times 0$ ". 57 (position angle $=-88^{\circ}$ ). Comparing with the CO (1-0) flux obtained by NRAO $12 \mathrm{~m}$ (Sanders et al. 1991),

\footnotetext{
${ }^{13}$ https://almascience.nrao.edu/sc/

${ }^{14}$ https://casaguides.nrao.edu/index.php/DataWeightsAndCombination
}

the recovered flux is $113 \pm 26 \%$, indicating that we recovered most (or probably all) of the $\mathrm{CO}(1-0)$ emission. The total $\mathrm{H}_{2}$ mass is $(4.1 \pm 0.5) \times 10^{9} M_{\odot}$ assuming one-fifth of the Milky Way CO-to- $\mathrm{H}_{2}$ conversion factor $\left(\alpha_{\mathrm{MW}}=4.3 \mathrm{M}_{\odot} /\left(\mathrm{K} \mathrm{km} \mathrm{s}^{-1}\right.\right.$ $\left.\mathrm{pc}^{2}\right)^{-1}$; Downes \& Solomon 1998; Bolatto et al. 2013b). When comparing with ACA-only CO (1-0) flux measured by König et al. $\left(2016 ; \sim 241 \mathrm{Jy} \mathrm{km} \mathrm{s}^{-1}\right.$ ), the recovered flux is $\sim 123 \%$. This difference might be due to the differences of mask shape to measure the $\mathrm{CO}$ flux in R.A., decl., and velocity axes (i.e., low surface brightness, high-velocity wings). Detailed information of the ACA and the $12 \mathrm{~m}$ array data is shown in Table 1 and the line information is listed in Table 2.

\section{Results}

The $u v$-matched CO integrated intensity, velocity field, and velocity dispersion maps are shown in Figure 2, except for the ACA-combined CO (1-0) image (Figure 3(a)). The channel maps are shown in the Appendix.

\section{1. uv-matched CO Intensity, Velocity Field, and Velocity Dispersion}

The $u v$-matched $\mathrm{CO}$ distributions of NGC 1614 can be divided into two regions: the starburst ring (central $590 \mathrm{pc}$ in radius) and an outer disk ( $\sim 3.3 \mathrm{kpc}$ in radius). The starburst ring is detected in X-ray, polycyclic aromatic hydrocarbon (HerreroIllana et al. 2014), Pa $\alpha$ (Alonso-Herrero et al. 2001), radio-toFIR continuum (Olsson et al. 2010; S16a), HCN (4-3), and $\mathrm{HCO}^{+}$(4-3) emissions (Imanishi \& Nakanishi 2013; Sliwa et al. 2014). Most of the $\mathrm{CO}$ emission in the starburst ring arises from the western side. The asymmetry in the starburst ring coincides with the radio-to-FIR continuum and the $\mathrm{HCO}^{+}(4-3)$ 


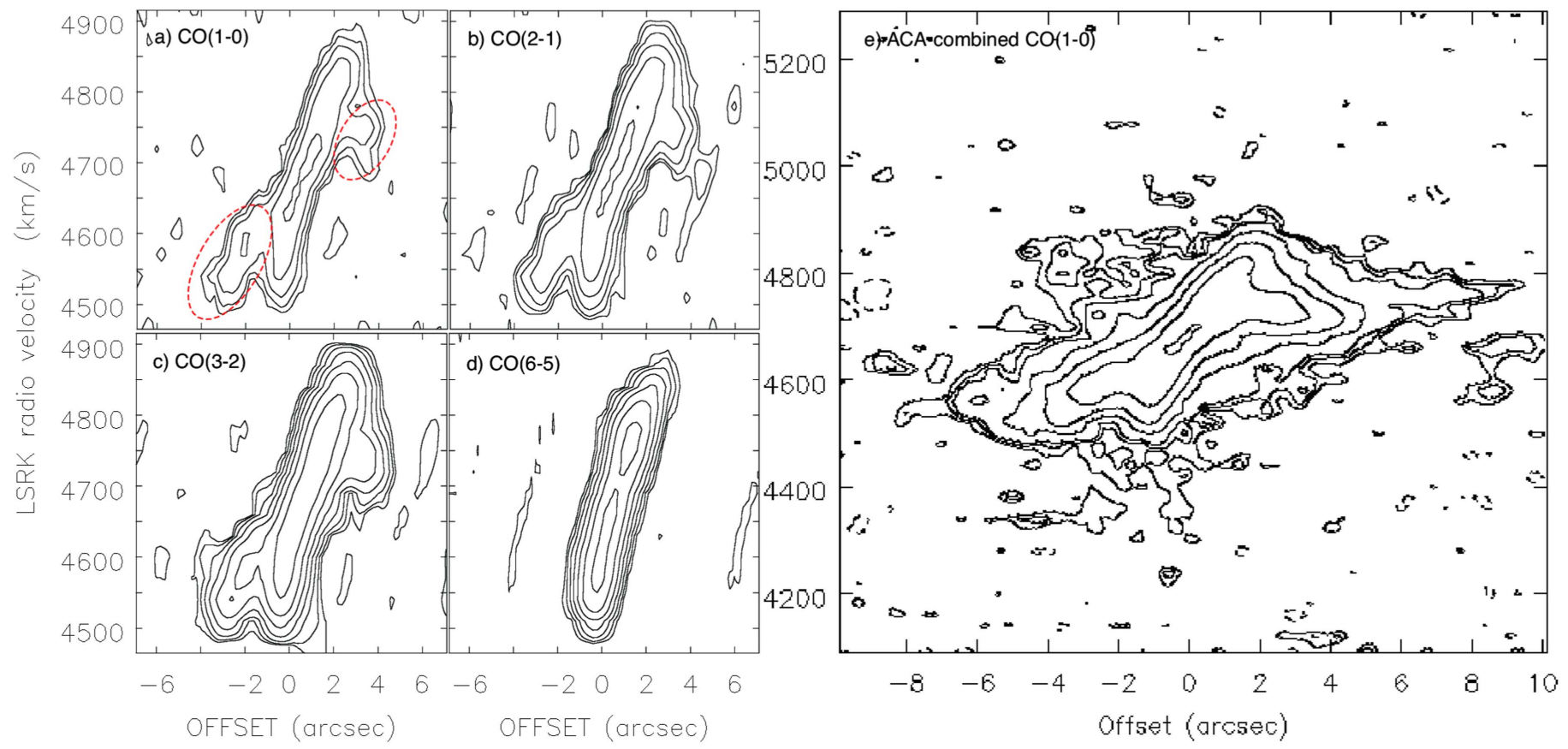

Figure 4. $(\mathrm{a} / \mathrm{b} / \mathrm{c} / \mathrm{d})$ Spatially averaged position-velocity diagram of each CO transition along the north-south direction (width $=13$ " 4 ) through the radio nucleus. The $n$th contours are at $2^{n} \sigma(n=1,2,3 \ldots) . \sigma$ is the noise rms listed in Table 2. Red circles show the non-circular motion detected in lower- $J$ transitions. (e) Spatially averaged position-velocity diagram of the ACA-combined $\mathrm{CO}(1-0)$ along the north-south direction. The contours are $0.5 \times(-2,2,3,4,8,16,32$, and 64$)$ mJy beam ${ }^{-1}$.

emissions. On the other hand, the outer disk is only detected in extended $\mathrm{Pa} \alpha$ and soft X-ray emissions (Herrero-Illana et al. 2014), indicating a site of moderate star formation relative to the ring (but we still have missing flux for extended low surface brightness $\mathrm{CO}(6-5)$ emission). All CO (1-0) peaks outside of the starburst ring coincide with the peaks detected in Pa $\alpha$ (Figure 1(c)).

The overall velocity structure of all of the $\mathrm{CO}$ transitions seems to be dominated by rotation. This is consistent with the velocity modeling with tilted concentric rings provided by Ueda et al. (2014). The velocity field and dispersion of the starburst ring smoothly connect to those of the outer disk, which indicates that the kinematics (e.g., position angle, inclination) are similar. We identify non-circular motions in a region with a length of $9^{\prime \prime}(\sim 3 \mathrm{kpc})$ that is extended from the north to the south of the disk (dashed lines in Figure 2(b)) in all $\mathrm{CO}$ images, except for $\mathrm{CO}(6-5)$. The apparent " $\mathrm{S}$ "-shape feature in the velocity field suggests the presence of noncircular motions (e.g., Kalnajs 1978, p. 113) in the region where the dust lane connects with the starburst ring (Figure 1(b)) or a warped disk (e.g., Centaurus A; Espada et al. 2012). The apparent characteristics are similar to the nearby barred spiral galaxy NGC 1097, which is a minor merger system (Lin et al. 2013).

We investigate the PVDs per the different $\mathrm{CO}$ transitions along the north-south direction with a width of 13 ". 4 and a length of 15 !" 0 through the nucleus (Figures $4(a)-(d)$ ). The width and length of these PVDs cover all of the $\mathrm{CO}$ emission (Figure 2). The PVDs show rigid rotation (i.e., starburst ring) in all CO transitions and the "S"-shape feature (dashed ellipses in Figure 4(a)), except for CO (6-5). The direction of the PVD cut is similar to the kinematical major axis determined from the CO (2-1) velocity field $\left(352^{\circ} \pm 1^{\circ}\right.$; Ueda et al. 2014).

While the large-scale kinematics can be explained by both circular and non-circular motion, the central region appears to show outflows. García-Burillo et al. (2015) found high-velocity CO (1-0) wings $\left(210 \mathrm{~km} \mathrm{~s}^{-1}<\left|v-v_{\text {sys }}\right|<450 \mathrm{~km} \mathrm{~s}^{-1}\right.$, where $v_{\text {sys }}=4763 \mathrm{~km} \mathrm{~s}^{-1}$ ) with the P.A. of $82^{\circ}$ in the central region. They suggested these components are a putative bipolar outflow with an outflow rate of $\sim 40 M_{\odot} \mathrm{yr}^{-1}$. The direction and the outflow rate is similar to those of the ionized gas outflow (Bellocchi et al. 2012). We did not detect the line wings in all $\mathrm{CO}$ transitions, although they are detected in the ACA-combined CO (1-0) image (see Section 3.2).

In Figure 5(a), we show the normalized radial distribution of the CO SLED of NGC 1614. The CO (6-5) emission is centrally concentrated, while the lower- $J$ CO transitions show an extended component (i.e., the outer disk). The peak radius shifts toward outside as the $\mathrm{CO}$ transition shifts to lower- $J$, showing a radial excitation gradient in the starburst ring (see Section 4.1). In Figure 5(b), as a comparison, we plot the normalized radial distribution of the radio-to-FIR SED of NGC 1614 with the same angular resolution and MRS (S16a). All of the continuum emission (synchrotron, free-free, and cold dust) are more compact than the lower- $J$ CO transitions, but the sizes are similar to the extent of the $\mathrm{CO}(6-5)$ emission. This suggests that the $\mathrm{CO}(6-5)$ is a better tracer of star-forming activity than lower- $J$ CO transitions (e.g., Xu et al. 2015). Although differences are seen in the spatial distribution between the transitions, there are no significant differences in the velocity profile within the central 2.33 radius, as shown in Figure 5(c), supporting that the rigid rotating component dominates the nuclear region.

\subsection{ACA 12 m Combined CO (1-0) Data}

We show the $\mathrm{CO}(1-0)$ image that is generated by combining the ACA data with the 12 array data in Figure 3(a). The global velocity field does not change significantly, although additional extended structures toward the north-east (redshifted) and 

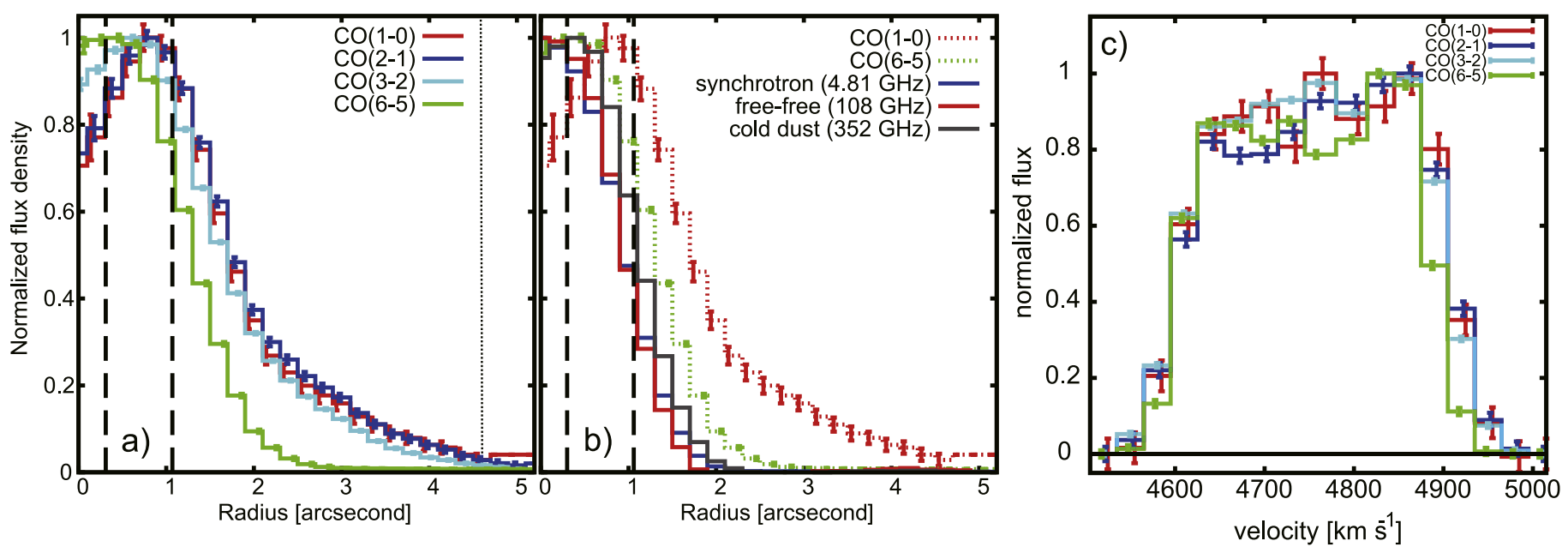

Figure 5. (a) Azimuthally averaged normalized radial distribution of each CO transition of NGC 1614 centered at the radio nucleus (Olsson et al. 2010). We only consider the statistical error. The dashed lines show the approximate inner and outer radii of the nuclear starburst ring (Xu et al. 2015). The dotted line shows the approximate outer radius of the field of view of the $\mathrm{CO}(6-5)$ data. (b) Comparison between each $\mathrm{CO}$ transition (dotted line) and each continuum emission (solid line; S16a). All of the data for the different CO transitions are convolved to the same resolution of $1{ }^{\prime \prime} 0$. (c) Normalized CO Spectra of NGC 1614 toward the central 4 !' 6 aperture.
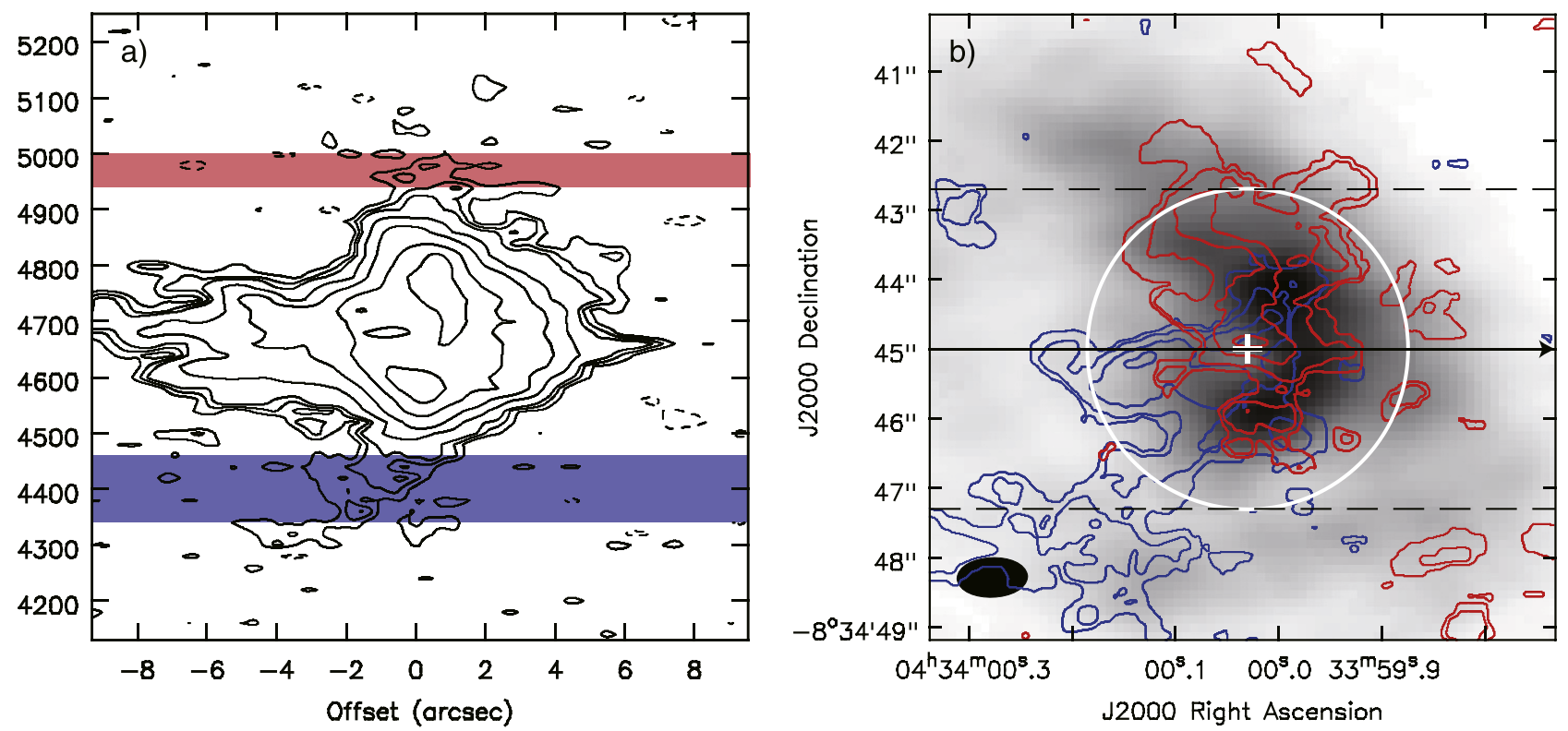

Figure 6. (a) Spatially averaged position-velocity diagram of the ACA-combined CO (1-0) data along the east-west direction (width $=4{ }^{\prime \prime} 6$ ). The contours are $0.5 \times\left(-2,2,3,4,8,16,32\right.$, and 64) $\mathrm{mJy}_{\text {beam }}{ }^{-1}$. This PVD only contains velocity components between the dashed lines in Figure 6(b). (b) Integrated intensity contour images of the high-velocity components (i.e., molecular outflow suggested by García-Burillo et al. 2015) overlaid on the ACA-combined CO (1-0) image (Figure 3(a)) with gray scale. The blue and red contours show blueshifted and redshifted components, respectively. The blue contours are $0.45 \times(0.16,0.32,0.64$, and 0.96$) \mathrm{Jy} \mathrm{beam}^{-1} \mathrm{~km} \mathrm{~s}^{-1}$, whereas the red contours are $0.32 \times(0.16,0.32,0.64$, and 0.96$) \mathrm{Jy} \mathrm{beam}{ }^{-1} \mathrm{~km} \mathrm{~s}^{-1}$. The white circle indicates the approximate outer edge of the starburst ring (König et al. 2013; Herrero-Illana et al. 2014; Xu et al. 2015; Saito 2016a).

south-west (blueshifted) appear. These arm-like structures are located along the optical dust lanes, as already found by the ACA-only CO (1-0) image (König et al. 2016) and $\mathrm{Pa} \alpha$ (Figure 1(c)). The low S/N high-velocity wings (Figures 3(b) and (c)) are consistent with the presence of a putative outflow suggested by García-Burillo et al. (2015).

We investigate the PVD of the ACA-combined image along the north-south (along the kinematical major axis with a width of 13!"4; Figure 4(e)) and east-west direction (along the kinematical minor axis with a width of 4".6; Figure 6(a)) through the radio nucleus (Olsson et al. 2010). Along the major axis, we found more extended components than the $u v$-clipped CO (1-0) image. The ACA data show features that are consistent with bar-induced inflow motions predicted by Iono et al. (2004). In contrast, as shown in the PVD along the minor axis, which emphasizes low surface brightness components within the 4!" 6 width, there are high-velocity components around the nuclear region. Since the high-velocity components show the same spatial and spectral distributions as the molecular gas outflow suggested by García-Burillo et al. (2015), we regard the high-velocity components as the molecular gas outflow. We constructed the integrated intensity image of the blueshifted (4340-4460 $\mathrm{km} \mathrm{s}^{-1}$ ) and redshifted $\left(4940-5000 \mathrm{~km} \mathrm{~s}^{-1}\right)$ outflows in Figure 6(b). The extracted velocity ranges contain high-velocity wings stronger than $3 \sigma$. The CO (1-0) outflow shows an extended blueshifted 

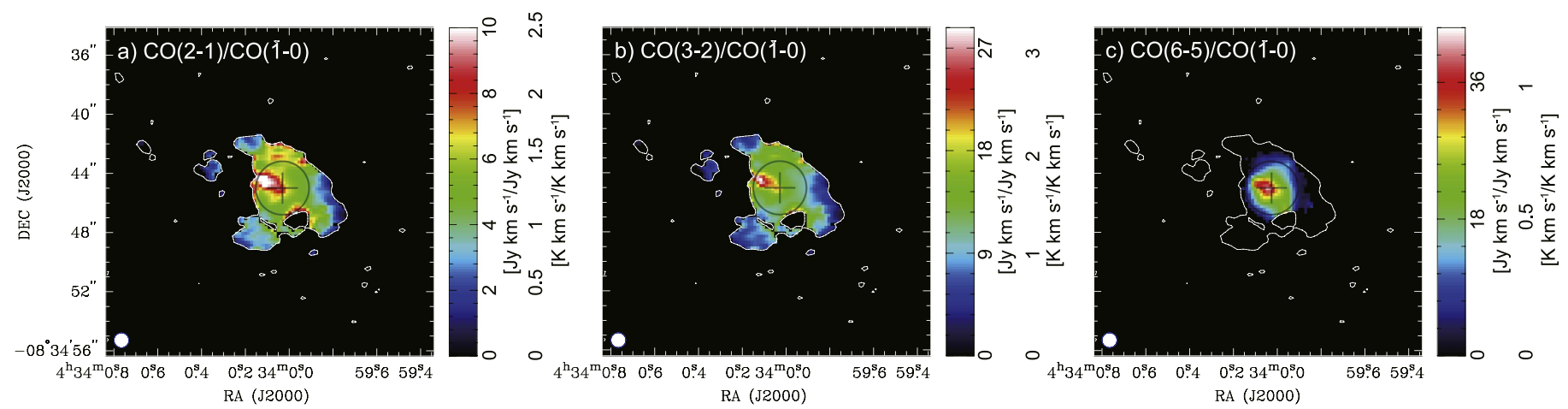

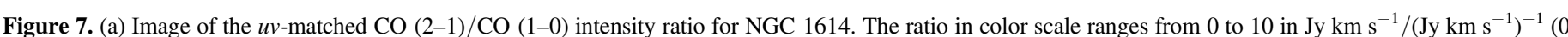

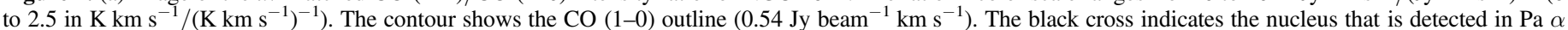

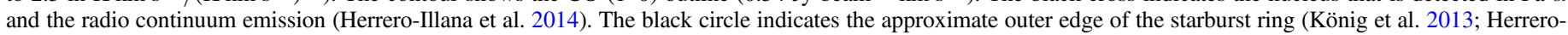

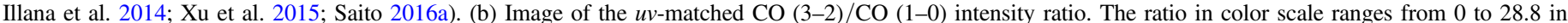

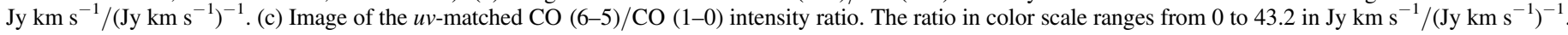

(redshifted) emission toward the south-east (north-west) direction. The molecular gas outflow seems to encompass the ionized gas outflow (Bellocchi et al. 2012). This picture is consistent with the molecular gas outflow entrained by the ionized gas outflow in the nearby starburst galaxy NGC 253 and M51 (Bolatto et al. 2013a; Querejeta et al. 2016).

\subsection{CO Line Ratios}

We show CO (2-1)/CO (1-0), CO (3-2)/CO (1-0), and $\mathrm{CO}(6-5) / \mathrm{CO}(1-0)$ line ratio images in Figures $7(\mathrm{a})-(\mathrm{c})$, respectively. We divide the integrated intensity maps (Figures 2(d), (g), and (j)) by the $\mathrm{CO}(1-0)$ integrated intensity map (Figure 2(a)) to make the line ratio maps. After that, we masked all the line ratio maps by using the $\mathrm{CO}(1-0)$ outline, which is shown in Figure 7 . The CO (2-1)/CO (1-0) and $\mathrm{CO}(3-2) / \mathrm{CO}(1-0)$ line ratios show higher values than the optically thick thermalized value, while the $\mathrm{CO}(6-5) /$ $\mathrm{CO}(1-0)$ line ratio is moderate. Those high $\mathrm{CO}(2-1) /$ $\mathrm{CO}(1-0)$ and $\mathrm{CO}(3-2) / \mathrm{CO}(1-0)$ line ratios indicate low optical depths for the CO (1-0) line (e.g., Downes \& Solomon 1998) and/or the presence of multiple-phase ISM (e.g., $\mathrm{CO}(3-2) / \mathrm{CO}(1-0)$ ratio in Arp 220; Rangwala et al. 2011). The peak position of each line ratio is located at $1^{\prime \prime}$ east of the nucleus. Since it coincides with the eastern part of a possible ionized and molecular gas outflow (Bellocchi et al. 2012; García-Burillo et al. 2015), the high line ratio component might be due to the interaction between the outflow and the starburst ring. Strong outflow can heat the surrounding ISM kinematically. This results in highly excited (i.e., low- $J$ depopulation) or highly turbulent (i.e., low optical depth) molecular gas around the shock interface, and thus high line ratios. A high $\mathrm{CO}(3-2) / \mathrm{CO}(1-0)$ ratio is also observed in the circumnuclear disk (CND) of Seyfert 2 galaxy NGC 1068 (see Section 7.2 of García-Burillo et al. 2014). The high ratio in the CND is located parallel to the bipolar jet, which may indicate that the CND is affected by the nuclear jet. A similar situation is found in the nuclear region of NGC 1614 (Figure 7), which may indicate outflow-ring interaction. However, further molecular gas and ionized gas observations are necessary to confirm this scenario quantitatively.

\section{CO SLED Modeling}

The CO SLEDs in U/LIRGs are often explained by a combination of CRDR, PDR, XDR, and MDR. However, especially for NGC 1614, previous studies suggested that contributions from CRDR and XDR are small or those models cannot reproduce observed quantities (see Herrero-Illana et al. 2014; Xu et al. 2014, in details), so we only focus on the effect of both PDR and MDR in this work.

\subsection{Single-phase RADEX Model}

We used the non-LTE radiative transfer code RADEX (van der Tak et al. 2007) and varied the parameters until the residuals between the observed line fluxes and the modeled line fluxes are minimized in a $\chi^{2}$ sense. Assuming a single-phase ISM (i.e., the gas physics can be represented by a single set of the excitation parameters), an expanding sphere geometry $\left(d v=200 \mathrm{~km} \mathrm{~s}^{-1}\right)$, the cosmic microwave background temperature $\left(T_{\mathrm{bg}}=T_{\mathrm{CMB}}=\right.$ $2.73 \mathrm{~K})$, and a $[\mathrm{CO}] /\left[\mathrm{H}_{2}\right]$ abundance $\left(3 \times 10^{-4}\right)$ that is similar to the standard value observed in Galactic molecular clouds (Blake et al. 1987), we derived the physical conditions of molecular gas. The upper state energies and the Einstein coefficients were taken from the Leiden Atomic and Molecular Database (Schöier et al. 2005). We varied the gas kinetic temperature within a range of $T_{\text {kin }}=10-300 \mathrm{~K}\left(d T_{\text {kin }}=1 \mathrm{~K}\right)$, the gas density of $n_{\mathrm{H}_{2}}=10^{2}-10^{7} \mathrm{~cm}^{-3}\left(d n_{\mathrm{H}_{2}}=10^{0.1} \mathrm{~cm}^{-3}\right)$, and the gas column density of $N\left(\mathrm{H}_{2}\right)=10^{20.5}-10^{23.5} \mathrm{~cm}^{-2}(\mathrm{dN}$ $\left(\mathrm{H}_{2}\right)=10^{0.1} \mathrm{~cm}^{-2}$ ). For the input parameters, we used the radial flux distribution of each $\mathrm{CO}$ line shown in Figure 5, which were radially binned at 0 .' 2 intervals.

We note that $T_{\mathrm{bg}}=T_{\mathrm{CMB}}$ is a reasonable assumption, although one might expect that, given the intense dusty star formation, a higher $T_{\text {bg }}$ is more suitable (i.e., $T_{\text {bg }} \sim T_{\text {dust }}$ ). We estimated the dust opacity at the starburst ring in Saito et al. (2016a) and found a low dust opacity $(<0.21)$ even at $690 \mathrm{GHz}$. Therefore, at this low frequency regime $(115-690 \mathrm{GHz})$, the background temperature cannot be as high as $T_{\text {dust }}$.

We show results of the RADEX modeling at the outer region contained within a radius of 2 " $8-3$ ". 0 in Figure 8 . Line ratios related to $\mathrm{CO}(6-5)$ show different distributions to lower- $J$ CO line ratios in the parameter planes, demonstrating the importance of the CO (6-5) data. The results as a function of radius are shown in Figures 9(a)-(c). The minimum reduced- $\chi^{2}$ of the central 1 ." 8 is relatively poor $(\geq 3.6)$, whereas that of the outer region $(r>1 . \prime 8)$ is $\lesssim 3.6$. Gas density is high $\left(\sim 10^{5} \mathrm{~cm}^{-3}\right)$ independently of the distance from the nucleus. On the other hand, both $T_{\text {kin }}(=20-70 \mathrm{~K})$ and $N\left(\mathrm{H}_{2}\right)$ $\left(=10^{22.0-22.9} \mathrm{~cm}^{-3}\right)$ decrease as the radius increases. Their 

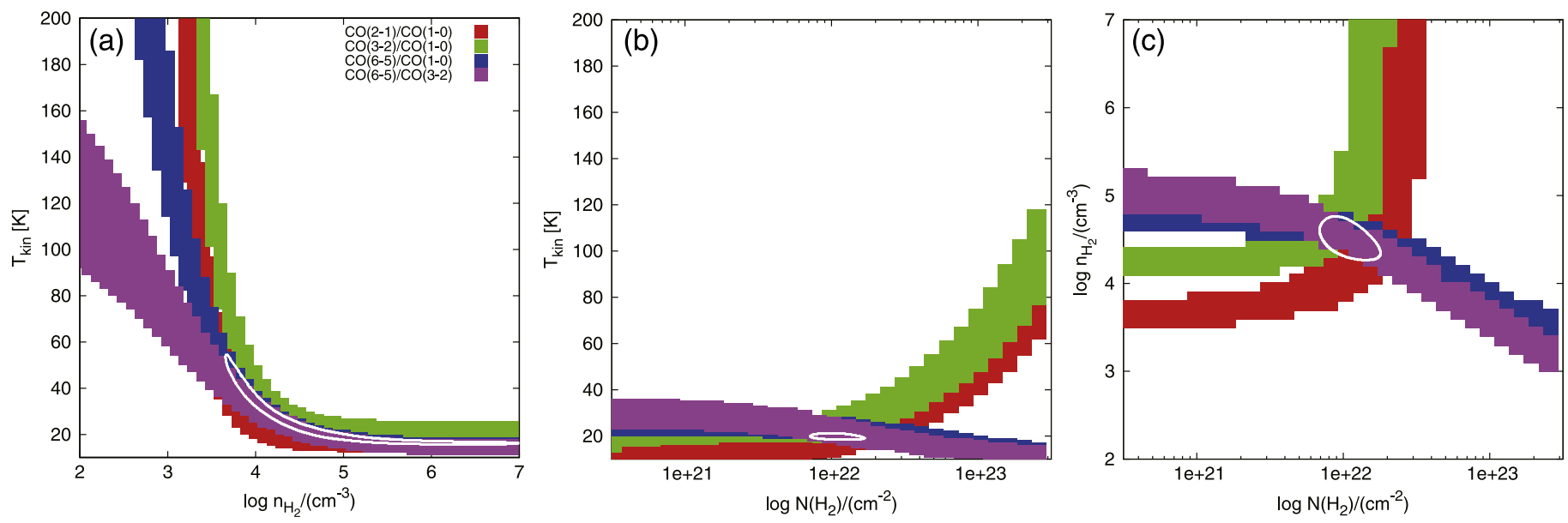

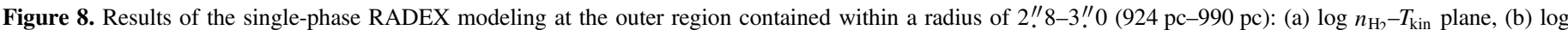

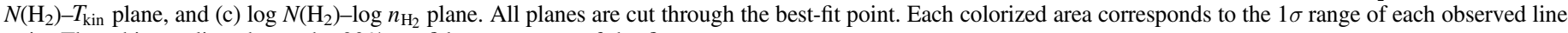
ratio. The white outline shows the $90 \%$ confidence contour of the fit.

peaks coincide with the radius of the starburst ring (100-350 pc; König et al. 2013; Xu et al. 2015). The derived $T_{\text {kin }}$ are about two times higher than the results of a similar modeling for NGC 1614 by Sliwa et al. (2014). This can be explained by the different spatial sampling between our analysis and theirs. In order to match the MRS between data sets, Sliwa et al. (2014) used single-dish data to compensate for the missing short baselines of their interferometric $\mathrm{CO}(2-1)$ and $\mathrm{CO}(3-2)$ data, while we clipped the inner $u v$ data at $45 \mathrm{k} \lambda$ (see Section 2).

We calculated the optical depth for each transition (Figure 10), yielding that $\tau_{\mathrm{CO}(1-0)}$ shows moderate optical depth $(\lesssim 1), \tau_{\mathrm{CO}(2-1)}$ and $\tau_{\mathrm{CO}(3-2)}$ are optically thick (1-10), and $\tau_{\mathrm{CO}(6-5)}$ becomes thin as the radius increases $\left(\tau_{\mathrm{CO}(6-5)}=8\right.$ to 0.1$)$. The derived $\tau_{\mathrm{CO}(1-0)}$ for NGC 1614 is consistent with that estimated for a mid-stage merger VV $114(\sim 1$; Saito et al. 2015), the central region of a close galaxy pair NGC 6240 (0.2-2; Iono et al. 2007), the overlap region of the mid-stage merger Arp 299 (0.5-1.5; Sliwa et al. 2012), and the local midstage Merger the Antennae (0.2-1.5; Zhu et al. 2003). This suggests that luminous mergers have a CO (1-0) line of moderate optical depths and a thick high- $J$ CO condition (e.g., Downes \& Solomon 1998).

We reconstructed the best-fit CO SLED from models in order to compare with the observational data (Figures 11(a) and (b)). The CO SLEDs in the outer disk $(>1$ "' 8$)$ show good agreement with the observations, while those in the starburst ring $(\lesssim 1$ !"8) systematically underestimated the CO (3-2)/ $\mathrm{CO}(1-0)$ and $\mathrm{CO}(6-5) / \mathrm{CO}(1-0)$ ratios. One possibility to explain the systematic difference is that the starburst ring of NGC 1614 has an additional warmer ISM, as suggested by Sliwa et al. (2014). The additional warm gas can move the modeled CO (3-2)/CO (1-0) and CO (6-5)/CO (1-0) ratios toward a higher value by fixing the lower- $J$ CO fluxes.

We note that the flux differences between the observations and the model are unlikely to be caused by dust extinction. Although some U/LIRGs show high dust opacities even in the submillimeter wavelengths (e.g., Rangwala et al. 2011; Wilson et al. 2014), the cold dust in the starburst ring of NGC 1614 is still optically thin at $691 \mathrm{GHz}(0.06-0.21$; S16a).

\subsection{Comparing with the Narayanan \& Krumholz Model}

We compare the observed CO SLED (Figure 11(a)) with the model of Narayanan \& Krumholz (2014), which can parameterize the CO SLED of galaxies by a power-law function of $\Sigma_{\text {SFR }}$. Using the $\Sigma_{\text {SFR }}$ derived by S16a, we reconstructed the CO SLED (Figure 11(c)). The $\Sigma_{\mathrm{SFR}}$ were derived by fitting the radial radio-to-FIR SEDs binned at 0 ". 2 intervals up to a 1." 8 radius assuming the absence of a strong or heavily obscured AGN. Each continuum image has the same angular resolution of $1 . \prime 0$ and MRS of 4." 6 as the CO images, so we can compare the observed CO SLEDs with the reconstructed CO SLEDs directly.

As clearly seen in Figures 11(a) and (c), this model is inconsistent with the observed CO SLEDs at the starburst ring $(<1$ "! 8$)$. The $\mathrm{CO}(3-2) / \mathrm{CO}(1-0)$ and $\mathrm{CO}(6-5) / \mathrm{CO}(1-0)$ ratios are also underestimated, as already described in Section 4.1. A multi-phase ISM model is one of the possible explanations. Because our analysis includes only four $\mathrm{CO}$ transitions and can only constrain a small number of free parameters, we will focus on a two-phase ISM model rather than a multiple-phase ISM (Section 5.4). The $\Sigma_{\mathrm{SFR}}$ used here is extinction-free because S16a used the low frequency continuum from 4.81 to $691 \mathrm{GHz}$. Thus, we can ignore the extinction effect in this comparison.

\subsection{PDR Model}

We used the PDR Toolbox (Kaufman et al. 2006), which can model the beam-averaged incident far-ultraviolet (FUV; $6 \mathrm{eV}<E<13.6 \mathrm{eV})$ radiation field intensity $\left(G_{0}\right.$ in the unit of $1.6 \times 10^{-3} \mathrm{erg} \mathrm{cm}^{-2} \mathrm{~s}^{-1}$ ) and PDR hydrogen nucleus density $\left(n \mathrm{~cm}^{-3}=n_{\mathrm{H}}+2 n_{\mathrm{H}_{2}}\right)$ from the flux density of each $\mathrm{CO}$ transition, in order to better understand the starburst activities of NGC 1614. We varied $n$ within the $90 \%$ confidence range of $2 n_{\mathrm{H}_{2}}$ derived by the single-phase RADEX $\left(d n=10^{0.25} \mathrm{~cm}^{-3}\right)$ and $G_{0}$ within a range of $10^{-0.50}-10^{6.50}$ $\left(d G_{0}=10^{0.25}\right)$. Here, we assumed that $n_{\mathrm{H}}$ is negligible compared to the $2 n_{\mathrm{H}_{2}}$ term because the central kiloparsecs of mergers are often dominated by the molecular phase (e.g., Iono et al. 2005; Kaneko et al. 2014). This assumption is consistent 

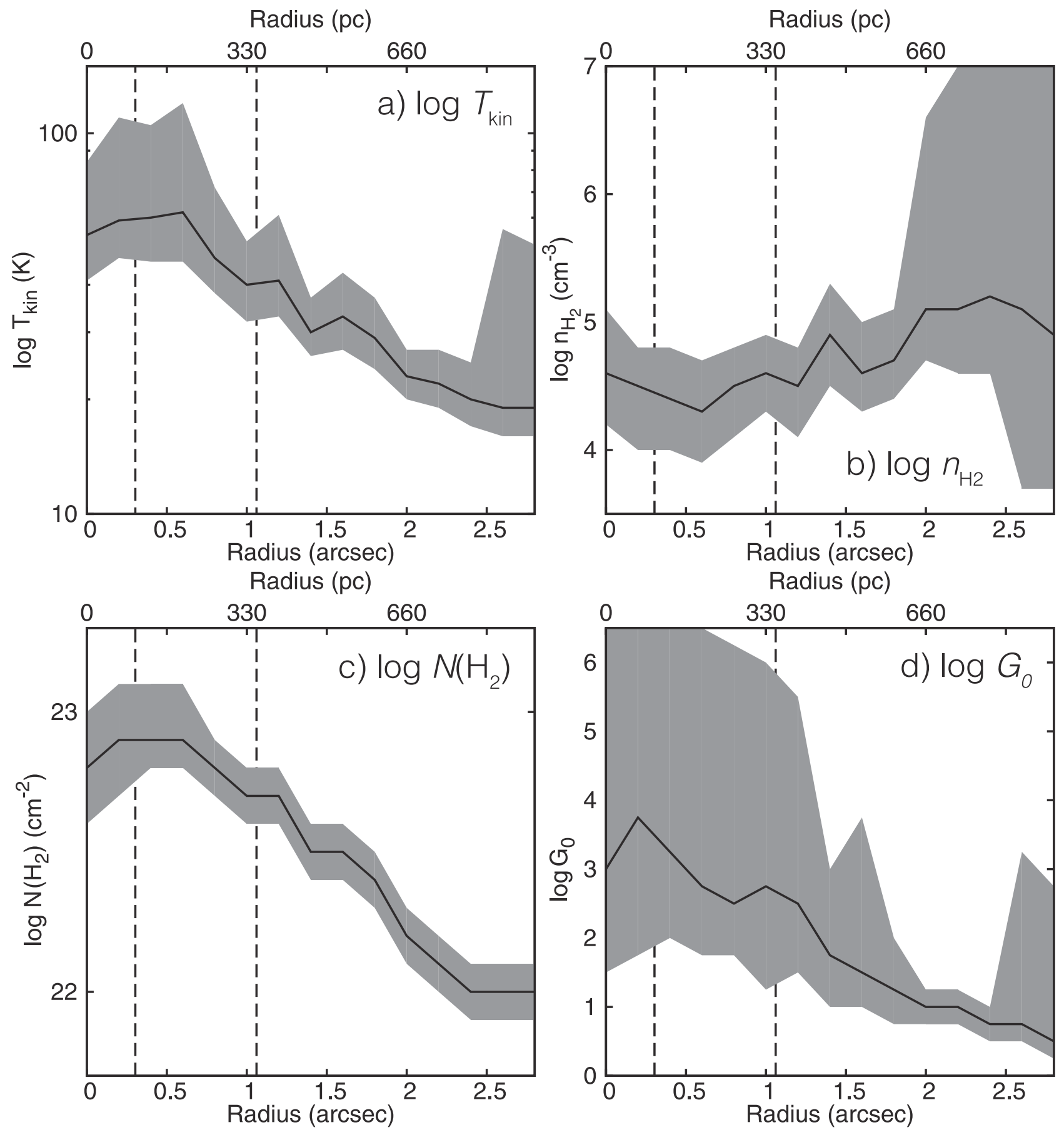

Figure 9. Results of the RADEX and PDR Toolbox modeling as a function of the radius; (a) kinetic temperature, (b) molecular gas density, (c) column density of molecular hydrogen, and (d) far-UV radiation field. The dashed lines show the approximate inner and outer radii of the nuclear starburst ring (Xu et al. 2015). The shaded areas show the $90 \%$ confidence.

with the compact $\mathrm{CO}$ distributions (Figure 2) and the extended arc-like H I distribution (Figure 1(a)) around the kiloparsec region of NGC 1614. The results are shown in Figure 9(d). The minimum reduced- $\chi^{2}$ is $\sim 1$ for both grids. The starburst ring has higher $G_{0}\left(>10^{1.5}\right.$, average $\left.=10^{2.7}\right)$ than the outer disk $\left(\lesssim 10^{1.5}\right.$, average $\left.=10^{0.9}\right)$. The average value of the whole galaxy is $\sim 10^{2.3}$.

In order to check the reliability of the derived $G_{0}$, we roughly estimate the averaged FUV radiation field on dust-obscured star-forming region using the prescription provided by
Papadopoulos et al. (2014),

$$
\begin{aligned}
G_{0}\left(L_{\mathrm{IR}}\right) \sim & \times 10^{2} \lambda_{*}(\mathrm{pc})\left(\frac{L_{\mathrm{IR}}}{10^{10} L_{\odot}}\right) \\
& \times\left(\frac{R_{\mathrm{SB}}}{100 \mathrm{pc}}\right)^{-3}\left[1-e^{-\left(R_{\mathrm{SB}} / \lambda_{*}\right)}\right],
\end{aligned}
$$

where $\lambda_{*}$ is the mean distance of FUV photons propagation before being absorbed and $R_{\mathrm{SB}}$ is the size of the starburst ring 


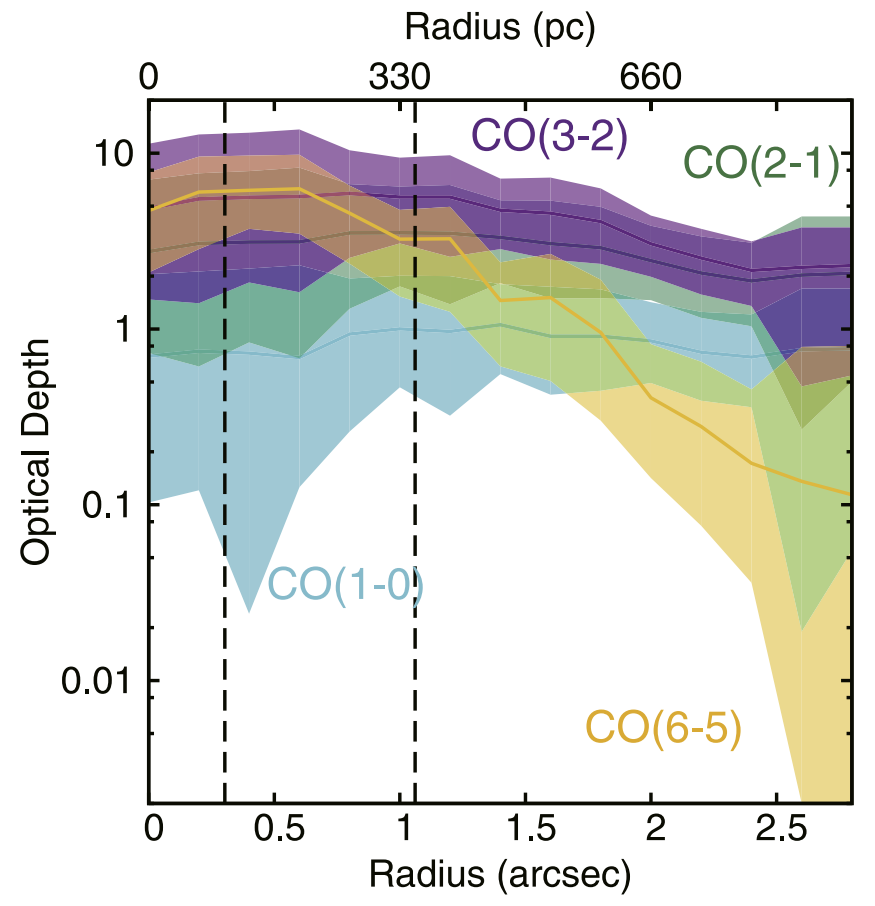

Figure 10. Optical depth of each CO transition as a function of the radius. The dashed lines show the approximate inner and outer radii of the nuclear starburst ring ( $\mathrm{Xu}$ et al. 2015). The shaded areas show the $90 \%$ confidence.

( $\sim 350$ pc; Xu et al. 2015). For the clumpy PDR model, $\lambda_{*}$ is $\lesssim$ 1 pc (Meixner \& Tielens 1993). The derived $G_{0}\left(L_{\mathrm{IR}}\right)$ is $\lesssim 10^{2.5}$, which is consistent with the average $G_{0}$ derived from the CO SLED.

\section{Discussion}

\subsection{The "S"-shape Velocity Field}

The "S"-shape morphology in the velocity field suggests the presence of a north-south bar or a warped disk morphology. It coincides with the leading edge of the main near-IR bar suggested by Olsson et al. (2010), so we suggest that the northsouth bar results in the "S"-shape non-circular motions (i.e., inflow along a bar) detected in the lower-J CO transitions. The presence of the cold gas inflow along the bar is also suggested by Olsson et al. (2010). In addition, analysis of $N$-body/SPH simulation performed by Iono et al. (2004) revealed that tidal interaction between gas-rich disks can produce gas flows directly toward the central regions due to the production of both transient arms and long-lived $m=2$ bars (i.e., bar instability). They also found that the inflowing gas is characterized by distinct diffuse gas clumps in the PVDs, while dense gas is only present in the central rigid rotating component. This simulation result is consistent with our observations including non-detection of the "S"-shape feature in the $\mathrm{CO}(6-5)$ image.

\subsection{Power Source of the Single-phase ISM}

We compare $T_{\text {kin }}$ and $G_{0}$ with $\Sigma_{\mathrm{SFR}}$, which is derived by modeling the radio-to-FIR SED (S16a) in Figure 12. There is a positive correlation with a correlation coefficient of $r=0.91$ between $\log \Sigma_{\text {SFR }}$ and $\log G_{0}$. This is a sensible result because both parameters are related to star formation. The relation between $\log \Sigma_{\mathrm{SFR}}$ and $\log T_{\text {kin }}$ also shows a positive correlation $(r=0.93)$. Using a PDR model described in Equation (3) of Papadopoulos et al. (2014) and assuming $T_{\text {kin }} \gtrsim T_{\text {dust }}$, which is found by PDR models (e.g., Hollenbach \& Tielens 1999), the derived $T_{\text {kin }}$ of $20-70 \mathrm{~K}$ corresponds to $\log G_{0}$ of $\lesssim 10^{1.4-4.6}$. This is consistent with the result of the CO SLED model described in Section 4.3. These models and a simple comparison indicates that the dusty starburst in NGC 1614 is enough to power the $\mathrm{CO}$ excitation up to $J_{\text {upp }}=6$ even if we just assume a single-phase molecular gas ISM for the starburst ring.

\subsection{Spatially Resolved CO-to- $\mathrm{H}_{2}$ Conversion Factor}

Molecular hydrogen mass is one of the most fundamental parameters to characterize galaxies in relation to their starbursts and AGNs. Although the galactic-scale CO (1-0) luminosity to $\mathrm{H}_{2}$ mass conversion factor $\left(M_{\mathrm{H}_{2}} / L_{\mathrm{CO}(1-0)}^{\prime} \equiv \alpha_{\mathrm{CO}(1-0)}\right)$ in nearby U/LIRGs has been well studied (see Bolatto et al. $2013 \mathrm{~b}$ for a review), the spatial properties are not fully understood.

We measure the $\alpha_{\mathrm{CO}(1-0)}$ as a function of radius using

$$
\alpha_{\mathrm{CO}(1-0)}=1.36 \frac{m_{\mathrm{H}_{2}} N\left(\mathrm{H}_{2}\right) S_{\text {ring }}}{L_{\mathrm{CO}(1-0)}^{\prime}} M_{\odot}\left(\mathrm{K} \mathrm{km} \mathrm{s}^{-1} \mathrm{pc}^{2}\right)^{-1} \text {, }
$$

where $m_{\mathrm{H}_{2}}$ is the mass of the hydrogen molecule, $N\left(\mathrm{H}_{2}\right)$ is the column density derived by RADEX (Section 4.1), $S_{\text {ring }}$ is the area of the concentric rings, and $L_{\mathrm{CO}(1-0)}^{\prime}$ is the $\mathrm{CO}(1-0)$ luminosity. We multiply by 1.36 to account for the helium abundance relative to hydrogen. The derived $\alpha_{\mathrm{CO}(1-0)}$ at the starburst ring is $0.2-0.5\left(\frac{3 \times 10^{-4}}{[\mathrm{CO}] /\left[\mathrm{H}_{2}\right]}\right)$, except for the central hole (central $0 . \prime 2$ radius), which yields $\sim 0.05\left(\frac{3 \times 10^{-4}}{[\mathrm{CO}] /\left[\mathrm{H}_{2}\right]}\right)$ (Figure 13). This is lower than $0.9-1.5\left(\frac{3 \times 10^{-4}}{[\mathrm{CO}] /\left[\mathrm{H}_{2}\right]}\right)$ at the starburst ring and $0.1\left(\frac{3 \times 10^{-4}}{[\mathrm{CO}] /\left[\mathrm{H}_{2}\right]}\right)$ at the central hole derived by Sliwa et al. (2014). The difference between both models can be explained by the presence of virialized giant molecular clouds (GMCs) and the different treatment of the missing flux. Assuming that GMCs in the central region of NGC 1614 are virialized, $\alpha_{\mathrm{CO}(1-0)}$ depends on gas density and kinetic temperature $\left(\alpha_{\mathrm{CO}(1-0)} \propto \rho^{0.5} T_{\mathrm{kin}}^{-1}\right.$, where $\rho$ is the gas density; Solomon \& Vanden Bout 2005; Bolatto et al. 2013b). As a consequence of the different missing flux treatment, the results of our RADEX modelings for the starburst ring are warmer $\left(27-120 \mathrm{~K}\right.$ and $\left.10^{3.9-5.3} \mathrm{~cm}^{-3}\right)$ than the results in Sliwa et al. (2014; 19-64 K and 103.0-6.5 $\mathrm{cm}^{-3}$; see also Section 4.1). Assuming the same gas density $(\rho)$ and beam filling factor for both models, $\alpha_{\mathrm{CO}(1-0)}$ derived from our model shall indeed be $\sim 2$ times lower than the Sliwa et al. (2014) value, indicating that both models are consistent.

We note that the treatment of the missing flux (i.e., how to achieve the same MRS for all transitions) should be different depending on the situation. When we have short-spacing data for "all" transitions, we should combine the data with data obtained with the compact configurations, which allows us to recover extended structures. In the case of this work, singledish or ACA data for several transitions are lacking, and thus we chose to clip the inner $u v$, making $\alpha_{\mathrm{CO}(1-0)}$ different from Sliwa et al. (2014). 


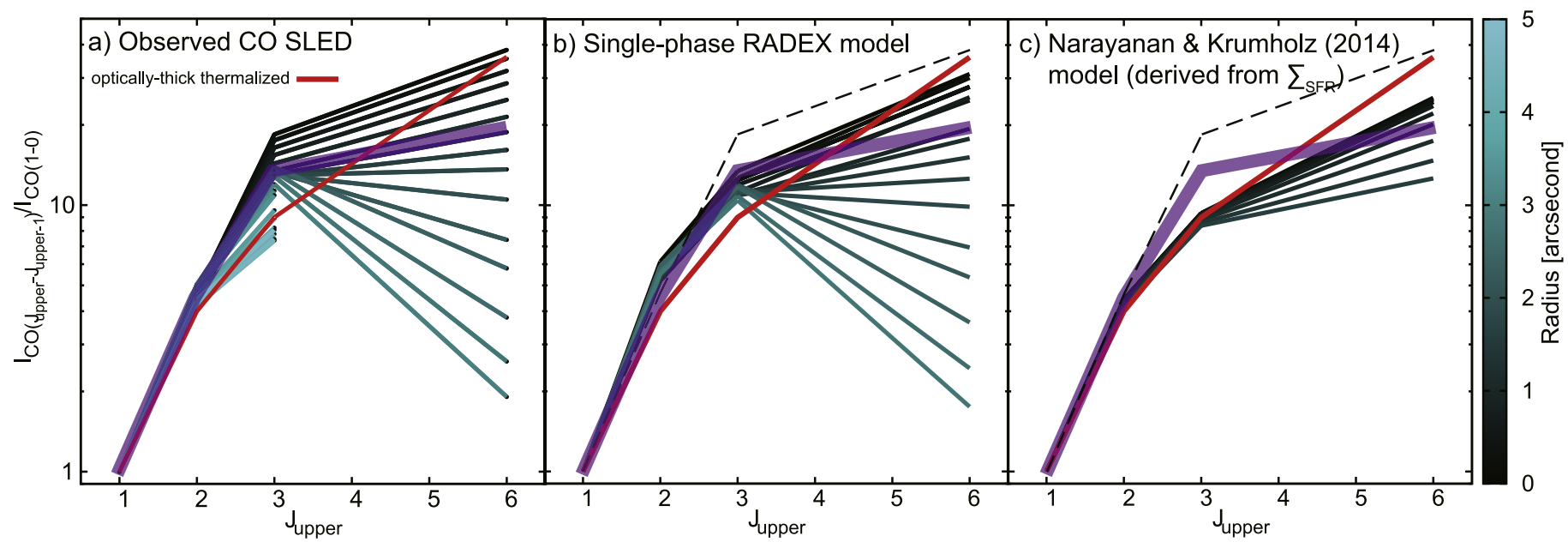

Figure 11. (a) Observed CO spectral line energy distributions (SLEDs) of NGC 1614 for concentric rings with the radius from $0 . ! 2$ to $5 !$. 0 . The color scale ranged from $0 . ! 0$ to 5 !. 0 ( 0. . 2 step from top to bottom lines) shows the radius of the concentric rings that are used for the photometry. The red line indicates a thermalized and optically thick CO SLED as a reference. (b) Modeled CO SLED reconstructed by the single-phase RADEX. The dashed line shows the observed CO SLED within the central 0 !' 2 radius. (c) Modeled CO SLED reconstructed from the Narayanan \& Krumholz (2014) model. The $\Sigma_{\text {SFR }}$ derived from the radio-to-FIR SED (S16a) is used as the input parameter.
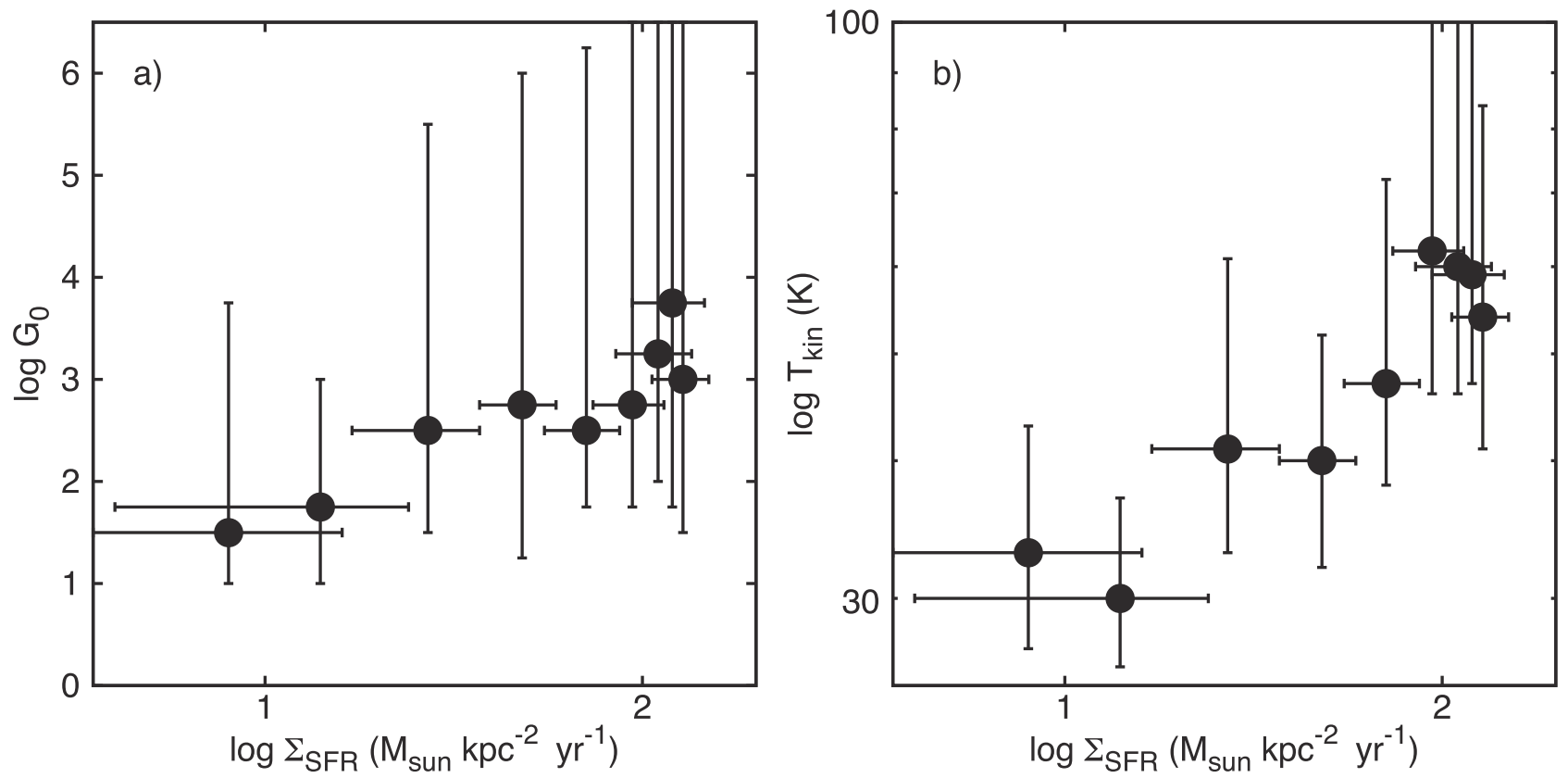

Figure 12. (a) Plot of the star formation rate surface density vs. the FUV radiation field at each concentric ring. The errors correspond to the $90 \%$ confidence. (b) Plot of the star formation rate surface density vs. the kinetic temperature at each concentric ring.

Comparing to local LIRGs, it is known that nearby spirals show larger $\alpha_{\mathrm{CO}(1-0)}$ (e.g., Bolatto et al. 2013b). Sandstrom et al. (2013) constructed $\alpha_{\mathrm{CO}(1-0)}$ as a function of galactocentric radius $\left(r_{25}=r / R_{25}\right.$, where $R_{25}$ is the $\mathrm{B}$-band isophotal radii at $25 \mathrm{mag} \operatorname{arcsec}^{-2}$ ) for 26 spiral galaxies and found lower $\alpha_{\mathrm{CO}(1-0)}$ at the galaxy centers $\left(r_{25}<0.2\right)$ by a factor of $1 \sim 5$ relative to their disks $\left(r_{25}=0.4-1.0\right)$. Using $R_{25}$ of $6.5 \mathrm{kpc}\left(\sim 20^{\prime \prime}\right)$ derived by $R_{25}=1.9 \times R_{\text {eff }}$ (Bellocchi et al. 2013) and $R_{\text {eff }}=3.44 \mathrm{kpc}$ (Ueda et al. 2014) for NGC 1614, the outer radius of the central hole, the starburst ring, and the CO disk of NGC 1614 are $r_{25} \sim 0.01,0.05$, and 0.14 , respectively (Figure 13). This indicates that NGC 1614 shows a similar radial $\alpha_{\mathrm{CO}(1-0)}$ trend to nearby spirals, but it has a more compact distribution and lower $\alpha_{\mathrm{CO}(1-0)}$ value than that of nearby spirals for a given galactocentric radius.
We calculate the conversion factor for $\mathrm{CO}(3-2)$ $\left(M_{\mathrm{H}_{2}} / L_{\mathrm{CO}(3-2)}^{\prime} \equiv \alpha_{\mathrm{CO}(3-2)}\right)$ and $\mathrm{CO}(6-5)\left(M_{\mathrm{H}_{2}} / L_{\mathrm{CO}(6-5)}^{\prime} \equiv\right.$ $\left.\alpha_{\mathrm{CO}(6-5)}\right)$ in Figure 14 assuming the single-phase ISM. The radial distribution of $\alpha_{\mathrm{CO}(3-2)}$ is almost the same as that of $\alpha_{\mathrm{CO}(1-0)}$, while the $\alpha_{\mathrm{CO}(6-5)}$ shows a steeper shape, except for the nuclear region $(<1$ !! 0$)$, which shows a similar value of $\alpha_{\mathrm{CO}(1-0)}$ because of the sub-thermalized conditions in the outer region.

\subsection{Two-phase Modeling for the Starburst Ring \\ 5.4.1. Two-phase ISM Model}

Herschel and other single-dish observations revealed that the CO SLED of nearby U/LIRGs can be represented by multiphase molecular gas ISM model (e.g., van der Werf et al. 2010; 


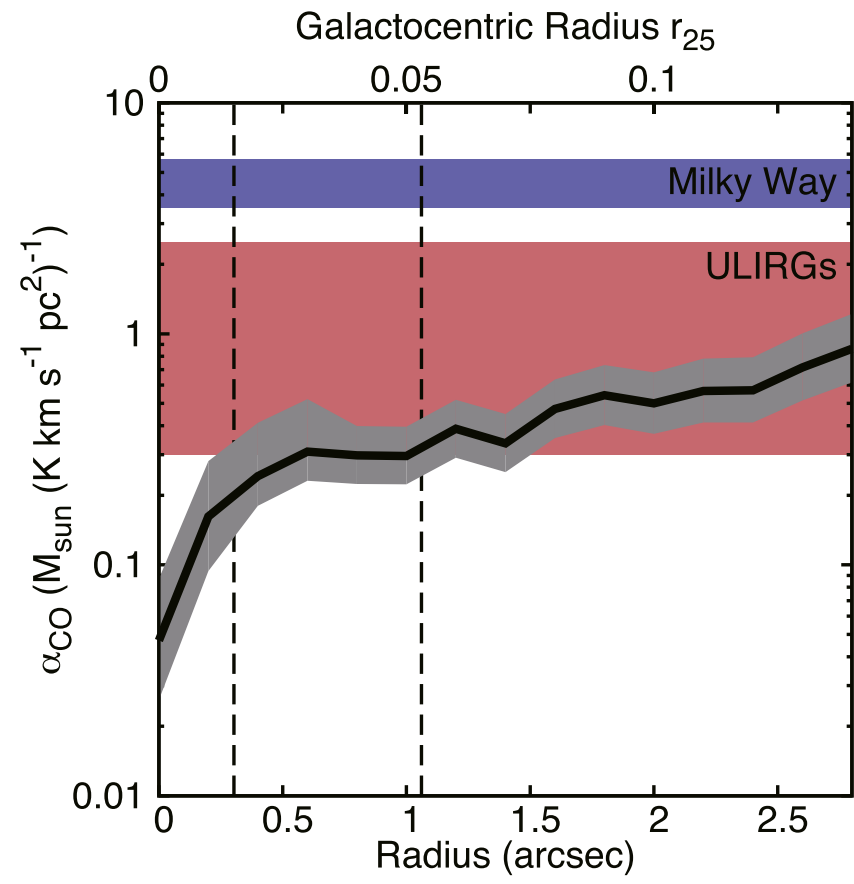

Figure 13. CO-to- $\mathrm{H}_{2}$ conversion factor $\left(=\alpha_{\mathrm{CO}(1-0)}=M_{\mathrm{H}_{2}} / L_{\mathrm{CO}(1-0)}^{\prime}\right)$ as a function of the radius derived by the single-phase RADEX model (i.e., $N\left(\mathrm{H}_{2}\right)$ ). The dashed lines show the approximate inner and outer radii of the nuclear starburst ring (Xu et al. 2015). The shaded areas show the $90 \%$ confidence. The blue and red regions show approximate $\alpha_{\mathrm{CO}(1-0)}$ for the Milky Way and ULIRGs (Bolatto et al. 2013b).

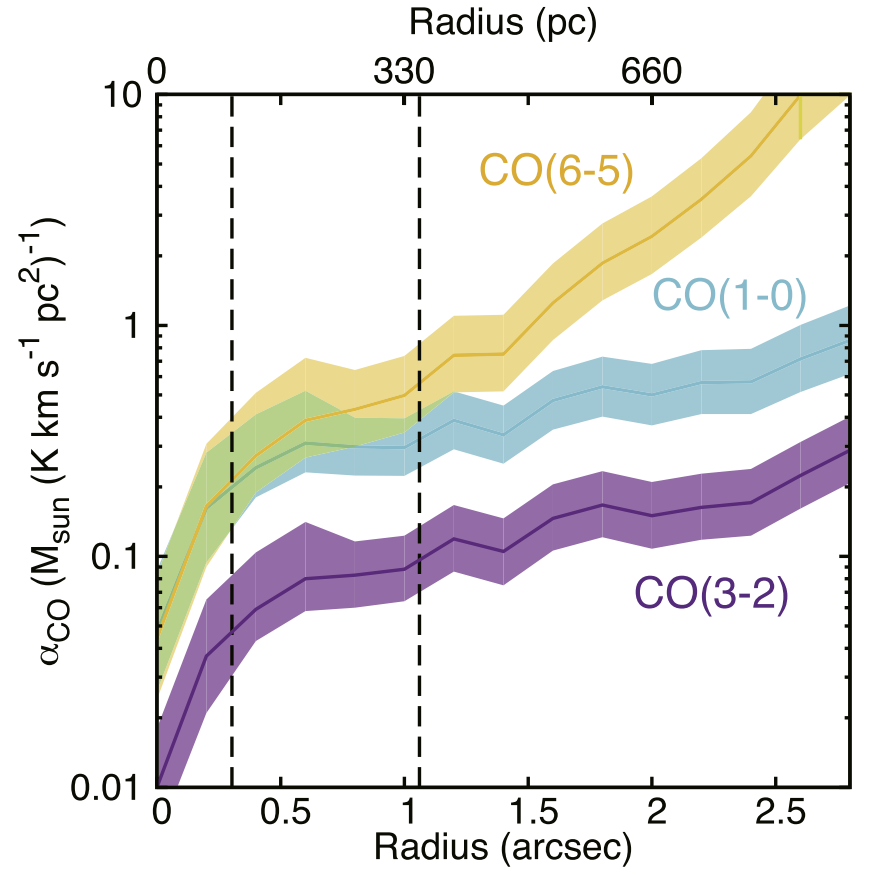

Figure 14. CO-to- $\mathrm{H}_{2}$ conversion factor for $\mathrm{CO}(1-0), \mathrm{CO}(3-2)$, and $\mathrm{CO}(6-5)$ lines as a function of the radius. The dashed lines show the approximate inner and outer radii of the nuclear starburst ring (Xu et al. 2015). The shaded areas show the $90 \%$ confidence.

Rangwala et al. 2011). Although the spatial properties of the CO SLED for U/LIRGs are unclear because of the limited number of high-resolution $\mathrm{CO}$ data, our high-resolution CO (1-0) and CO (2-1) observations, as well as the archival

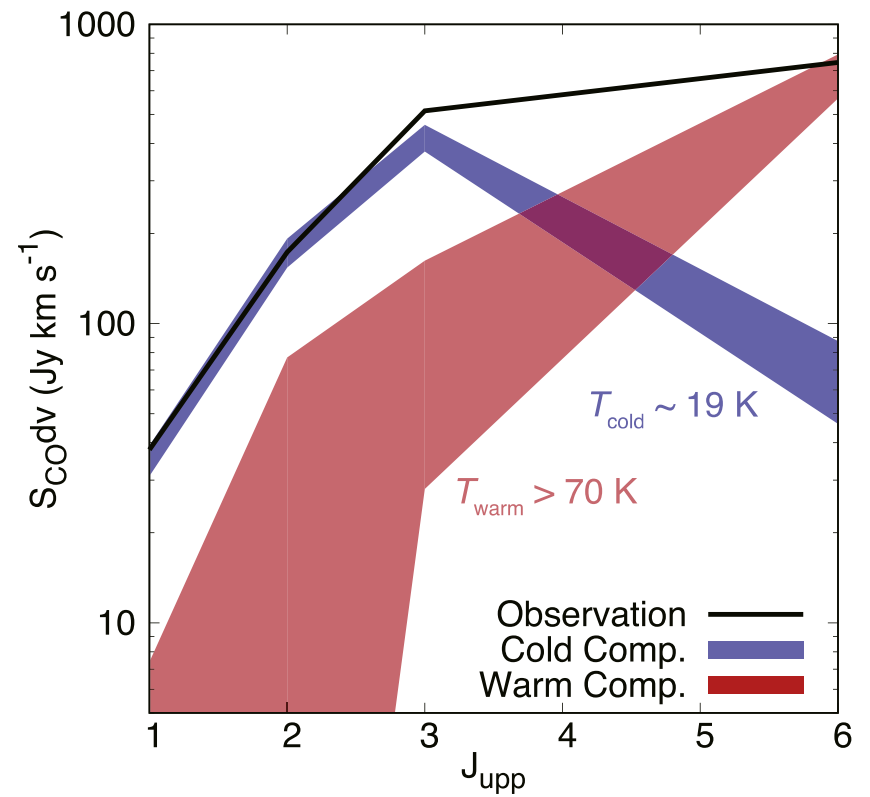

Figure 15. Two-phase CO SLED model. Dark line shows the observed CO SLED toward a central 1". 8 (594 pc) radius. The red and blue areas show the $90 \%$ confidence range of the warm and cold components, respectively. The areas cover the results from grids $\mathrm{A}, \mathrm{B}$, and $\mathrm{C}$.

CO (3-2) and CO (6-5) data, allow us to study this for a LIRG for the first time.

Before discussing the two-phase molecular gas ISM in NGC 1614, we note that the dust ISM in the starburst ring can be described by warm and cold components. Pereira-Santaella et al. (2015) reported the presence of a warm dust component in the starburst ring $(\sim 110 \mathrm{~K})$ revealed by modeling of the mid-IR emission, although there is also a cold dust component $(\sim 35 \mathrm{~K}$; $\mathrm{Xu}$ et al. 2015). In an ISM where the gas and dust are intermingled, the molecular gas component separates into a warm component of $T_{\text {warm }} \sim 110 \mathrm{~K}$ and a cold component of $T_{\text {cold }} \sim 35 \mathrm{~K}$. This can be addressed by RADEX with simple assumptions.

In order to model the warm and cold gas components, we minimize the number of free parameters by fixing the cold gas component using the observed line ratios at the outer region contained within a radius of 2 ". $8-3$ ". 0 . This region shows a good agreement with a single-phase model with a temperature of $T_{\text {cold }} \sim 19 \mathrm{~K}$ (Figure 9(a)). Here, we assume that cold molecular clouds, which dominate the outer region, dominate the cold gas component in the starburst ring. We calculate the contribution of the warm gas component to the total CO luminosity by using

$$
r=\frac{L_{\mathrm{CO}(2-1), \mathrm{warm}}^{\prime}}{L_{\mathrm{CO}(2-1), \mathrm{obs}}^{\prime}},
$$

where $L_{\mathrm{CO}(2-1) \text {,warm }}^{\prime}$ is the $\mathrm{CO}(2-1)$ luminosity of the warm gas component and $L_{\mathrm{CO}(2-1), \text { obs }}^{\prime}$ is the observed $\mathrm{CO}(2-1)$ luminosity within the central 1 !" 8 radius.

In the fitting routine, we varied $r$ within a range of $0-0.009$ $(d r=0.001), 0.01-0.09(d r=0.01)$, and 0.1-0.9 $(d r=0.1)$ to estimate the CO luminosities of the warm gas and cold gas components. Then, we varied the warm gas kinetic temperature within a range of $T_{\text {warm }}=10-800 \mathrm{~K}\left(d T_{\text {warm }}=10 \mathrm{~K}\right)$ and the warm gas column density of $N\left(\mathrm{H}_{2}\right)_{\text {warm }}=10^{19}-10^{24} \mathrm{~cm}^{-2}(d N$ $\left.\left(\mathrm{H}_{2}\right)_{\text {warm }}=10^{0.1} \mathrm{~cm}^{-2}\right)$. We fixed $[\mathrm{CO}] /\left[\mathrm{H}_{2}\right]\left(=3 \times 10^{-4}\right)$ and the gas density of the warm gas component as 
$n_{\text {warm }}=10^{2.0}$ (grid A), $10^{3.0}$ (grid B), and $10^{4.0} \mathrm{~cm}^{-3}$ (grid C). The results are shown in Figure 15. The minimum $\chi^{2}$ are 1.30 (grid A), 1.05 (grid B), and 0.85 (grid C), which are smaller than the $\chi^{2}$ values of the single-phase modeling for the starburst ring $(r<1$ !" 8$)$. The $90 \%$ confidence ranges of $T_{\text {warm }}$ are $300-370,>250$, and $70-450 \mathrm{~K}$, whereas those of $N$ $\left(\mathrm{H}_{2}\right)_{\text {warm }}$ are $>10^{23.7},>10^{22.9}$, and $10^{23.0-23.6}$ for the grids $\mathrm{A}, \mathrm{B}$, and $\mathrm{C}$, respectively. The $90 \%$ confidence ranges of $r$ are $<0.1$ (best-fit $=0$ ), $<0.8$ (best-fit $=0$ ), and $<0.6$ (best-fit $=0.003$ ) for the grids $\mathrm{A}, \mathrm{B}$, and $\mathrm{C}$, respectively, indicating less contribution of the warm gas component to the lower- $J \mathrm{CO}$ fluxes. This two-phase ISM model in NGC 1614 is similar to that found in the nearby ULIRG Arp 220, showing that a warm gas component has almost no contribution to $\mathrm{CO}$ lines lower than $J=3-2$ (Rangwala et al. 2011).

Using the PDR model (Section 4.3) assuming $n=$ $10^{2.0-5.0} \mathrm{~cm}^{-3}$, we find that the FUV field $\left(G_{0}\right)$ of the inferred warm gas component is higher than $10^{6.5}$. Considering the relatively low $\Sigma_{\text {SFR }}(\mathrm{S} 16 \mathrm{a})$ and the absence of a heavily obscured AGN, such an extreme radiation field is unreasonable for NGC 1614.

In summary, the starburst ring of NGC 1614 can be described by a two-phase molecular gas ISM with a cold component of $\sim 19 \mathrm{~K}$ (dominated by normal GMCs) and a warm component of $>70 \mathrm{~K}$ (warm ISM directly related to powerful activities). UV radiation produced by star-forming activities (i.e., clumpy PDRs) are not possible heating sources of the warm gas. We note that these models are consistent with a two-phase dust ISM $(\sim 35$ and $\sim 110 \mathrm{~K})$ of the starburst ring (Pereira-Santaella et al. 2015; Xu et al. 2015) and multi-phase models for other U/LIRGs (e.g., van der Werf et al. 2010; Rangwala et al. 2011).

\subsubsection{Power Source of the Warm Gas}

The observed high- $J$ CO fluxes are systematically higher than those in the Narayanan \& Krumholz model (Section 4.2). Also, FUV radiation heating due to the observed star-forming activities cannot reproduce the possible warm gas component (Section 5.4.1) assuming the two-phase model. Here, we employ the mechanical heating (i.e., shock excitation) as an alternative mechanism to power the warm gas. We found the largest values of the $\mathrm{CO}(2-1) / \mathrm{CO}(1-0)$ and $\mathrm{CO}(3-2) /$ CO (1-0) ratios ( $\sim 10$ and $\sim 27$, respectively) along with the putative outflow as described in Section 3.3, which are similar to what has been observed in the nearby Seyfert galaxy NGC 1068 ( 10 and $\sim 35$, respectively at the E-knot; Viti et al. 2014). NGC 1068 has a CND, which is thought to be heated by the nuclear jet (García-Burillo et al. 2014). The similarity between these galaxies may suggest that in the central 1."8 of NGC 1614 the CO emission is significantly affected by the kinematical interaction between the starburst ring and the putative outflow. The outflow can heat the starburst ring kinematically, producing highly excited molecular gas components around the shocked region. This scenario is consistent with the detection of $\mathrm{H}_{2} 1-0 \mathrm{~S}(1)$ line within the central 1". 8 radius (Alonso-Herrero et al. 2001; Kotilainen et al. 2001), suggesting that nearly one-third of the total near-IR $\mathrm{H}_{2}$ is produced by shocks. Stellar feedback such as supernova explosions and stellar winds are also possible sources of mechanical heating. Therefore, we compare the kinetic luminosity of the outflow and the degree of stellar feedback with the total CO luminosity of the warm gas.
We derive the kinetic luminosity from the supernovae $\left(L_{\mathrm{SN}}\right)$ using (Maloney 1999),

$$
L_{\mathrm{SN}} \sim 3 \times 10^{43}\left(\frac{\nu_{\mathrm{SN}}}{1 \mathrm{yr}^{-1}}\right)\left(\frac{E_{\mathrm{SN}}}{10^{51} \mathrm{erg}}\right) \mathrm{erg} \mathrm{s}^{-1},
$$

where $\nu_{\mathrm{SN}}$ is the supernova rate and $E_{\mathrm{SN}}$ is the energy of a supernova $\left(\sim 10^{51} \mathrm{erg}\right.$; Rangwala et al. 2011). Using the $\nu_{\mathrm{SN}}$ of $\sim 0.9$ (Rosenberg et al. 2012), we obtain $L_{\mathrm{SN}}$ of $\sim 2.7 \times$ $10^{43} \mathrm{erg} \mathrm{s}^{-1}$. Assuming the energy from stellar winds is similar to supernovae (McCray \& Kafatos 1987), the total kinetic luminosity from the stellar feedback is $\sim 5.4 \times 10^{43} \mathrm{erg} \mathrm{s}^{-1}$. On the other hand, the kinetic luminosity from the putative outflow is $\sim 2.5 \times 10^{42} \mathrm{erg} \mathrm{s}^{-1}$ (García-Burillo et al. 2014). Comparing with the inferred CO luminosity of the warm gas component $\left(\sim 1.2 \times 10^{41} \mathrm{erg} \mathrm{s}^{-1}\right.$; sum up to $\left.J_{\text {upp }}=23\right)$, both the outflow and the stellar feedback are possible heating sources of the warm gas component, even when we adopt a low-energy injection efficiency of $\lesssim 5 \%$. Although the molecular outflow is a possible source in terms of the energy, the high-resolution $\mathrm{CO}(6-5)$ image (Xu et al. 2015) shows that the distribution is closely related to the starburst ring, not the outflow (Figure 6(a)). This indicates that stellar feedback is more likely to be the heating source for higher- $J$ CO rather than the putative molecular outflow when we consider both energy and spatial $\mathrm{CO}(6-5)$ distribution. We note that the mechanical energy is also a possible heating source in Arp 220 (Rangwala et al. 2011), NGC 6240 (Meijerink et al. 2013), and other U/LIRGs (Greve et al. 2014).

\subsection{Balance of Molecular Gas in the Starburst Ring}

In order to evaluate the effect of the molecular gas inflow, we estimate molecular gas inflow rate (IFR) using

$$
\mathrm{IFR}=\frac{M_{\text {in }} v_{\text {in }}}{r_{\text {in }}} M_{\odot} \mathrm{yr}^{-1},
$$

where $M_{\text {in }}$ is the inflowing $\mathrm{H}_{2}$ mass, $v_{\text {in }}$ is the inflowing velocity, and $r_{\text {in }}$ is the distance from the starburst ring (e.g., Combes et al. 2013). Assuming the inflowing component is along the gas disk, Equation (5) can be calculated as follows.

$$
\begin{aligned}
\text { IFR } & =M_{\text {in }}\left(\frac{v_{\text {los }}}{\cos \theta}\right)\left(\frac{\sin \theta}{r_{\text {proj }}}\right) \\
& \sim 54\left(\frac{\tan \theta}{\tan 33^{\circ}}\right)\left(\frac{\alpha_{\mathrm{CO}}}{0.2 \alpha_{\mathrm{MW}}}\right) M_{\odot} \mathrm{yr}^{-1},
\end{aligned}
$$

where $v_{\text {los }}$ is the line-of-sight velocity relative to the rotation velocity at the projected distance $\left(r_{\text {proj }}\right), \theta$ is the inclination of the disk, and $\alpha_{\mathrm{MW}}$ is the $\mathrm{CO}-$ to- $\mathrm{H}_{2}$ conversion factor for the Galaxy (=4.3; Bolatto et al. 2013b). Here, we adopt $\theta$ of $33^{\circ}$ and $\alpha_{\mathrm{CO}}$ of $0.2 \alpha_{\mathrm{MW}}$, which are the same values used to derive the molecular gas outflow rate (OFR; García-Burillo et al. 2015). We estimate the flux density of the inflowing gas components using Figure 4(e). The northern (southern) inflowing component has 25.8 (19.3) $\mathrm{Jy} \mathrm{km} \mathrm{s}^{-1}$, which corresponds to $M_{\text {in }}$ of $10^{8.40}\left(\frac{\alpha_{\mathrm{CO}}}{0.2 \alpha_{\mathrm{MW}}}\right)\left(10^{8.27}\left(\frac{\alpha_{\mathrm{CO}}}{0.2 \alpha_{\mathrm{MW}}}\right)\right) M_{\odot} \cdot v_{\mathrm{los}}$ and $r_{\text {proj }}$ of the northern (southern) component are $80(40) \mathrm{km} \mathrm{s}^{-1}$ and $500(500) \mathrm{pc}$, respectively. The derived IFR is $54 M_{\odot} \mathrm{yr}^{-1}$ 

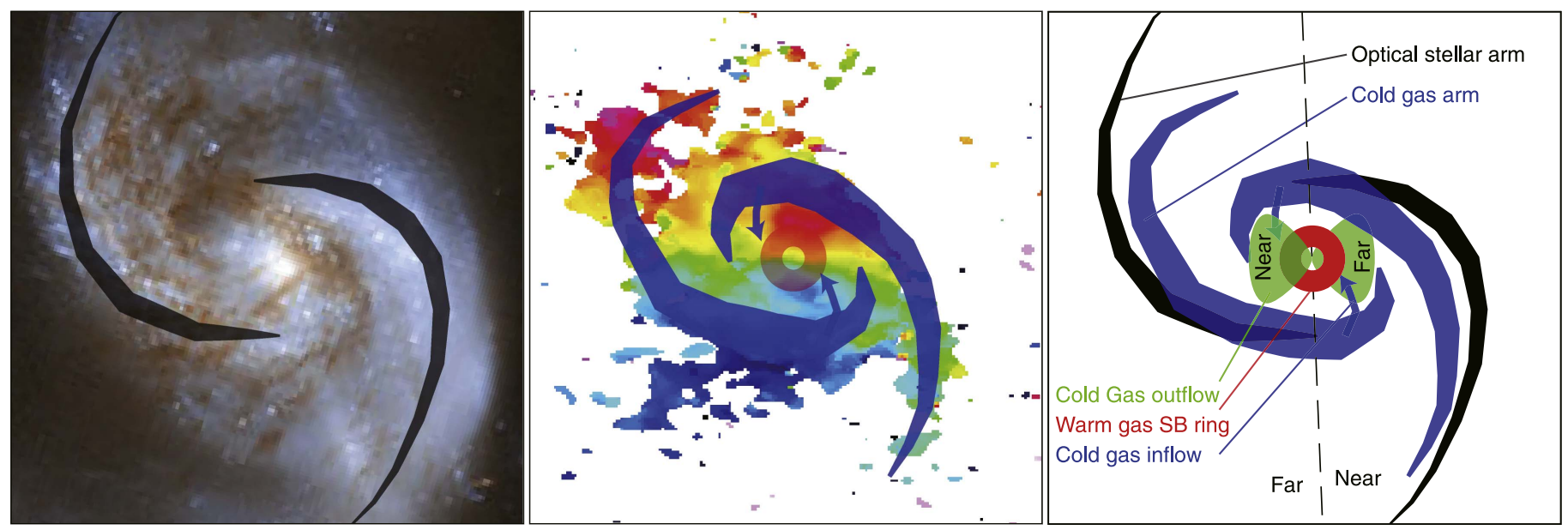

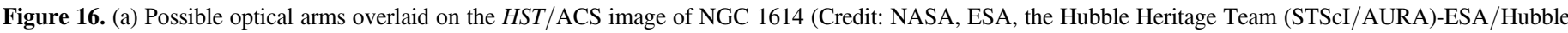

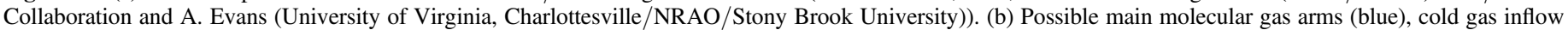
(blue arrows), and the starburst ring detected in CO (6-5) (red; Xu et al. 2015). (c) Schematic illustration of the possible geometry of NGC 1614.

with the uncertainty as a factor of three, which is mainly due to the uncertainties of $v_{\text {los }}$ and $r_{\text {proj. }}$.

Comparing the SFR in the starburst ring $\left(32.8 M_{\odot} \mathrm{yr}^{-1}\right.$; Xu et al. 2015) and OFR $\left(40 \pm 12 M_{\odot} \mathrm{yr}^{-1}\right.$; García-Burillo et al. 2015), and assuming that the IFR, OFR, and SFR continue constantly, we can investigate the balance between molecular gas input and output in the starburst ring of NGC 1614.

$$
\frac{\mathrm{IFR}}{\mathrm{SFR}+\mathrm{OFR}} \sim \begin{cases}0.7 & \left(\theta=33^{\circ}, \alpha_{\mathrm{CO}}=0.2 \alpha_{\mathrm{MW}}\right) \\ 1.2 & \left(\theta=33^{\circ}, \alpha_{\mathrm{CO}}=\alpha_{\mathrm{MW}}\right) .\end{cases}
$$

Independent of the adopted $\alpha_{\mathrm{CO}}$, the molecular gas input and output are roughly balanced, indicating that NGC 1614 can sustain both star-forming activities and an outflow in the starburst ring until exhausting the molecular gas reservoir in the starburst ring and the outer region via bar-driven inflow. The total molecular gas depletion time due to star formation and outflow $\left(=M_{\mathrm{H}_{2}} /(\mathrm{SFR}+\mathrm{OFR})\right)$ ranges from $17.7 \pm 4.6 \mathrm{Myr}$ $\left(\alpha_{\mathrm{CO}}=\alpha_{\mathrm{MW}}\right)$ to $56.6 \pm 9.3 \mathrm{Myr}\left(\alpha_{\mathrm{CO}}=0.2 \alpha_{\mathrm{MW}}\right)$. This is consistent with the typical duration timescale of U/LIRG phase predicted by numerical merger simulations (dozens of Myr; Saitoh et al. 2009; Teyssier et al. 2010). The luminous merger remnant NGC 1614 may quench itself until the next dozens of Myr due to depletion of the cold molecular gas reservoir (if the molecular outflow and star formation continue constantly).

\subsection{Central Gas Geometry of NGC 1614}

We summarize all results provided by this work and previous studies in a simple schematic picture (Figure 16). We used a position angle of $\sim-352^{\circ}$ and an inclination of $\sim 36^{\circ}$ as estimated by Ueda et al. (2014) for the cold gas disk and the starburst ring. This is consistent with the results provided by García-Burillo et al. (2015). We found " $\mathrm{S}$ "-shape non-circular motions along the leading edge of the north-south near-IR bar in the lower- $J$ CO velocity fields and PVDs. The near-IR bar connects to the near-IR spiral arms (Olsson et al. 2010). Moreover, the northern part of the molecular bar was detected in SMA CO (2-1) observations (dashed line in Figure 3 of König et al. 2013). König et al. (2016) also suggested that the northern molecular gas components are diffuse gas clouds that are now funneled into the nuclear region, although the flowing direction is different from the picture shown in Figure 16. The central starburst ring (SFR $\sim 32.8 M_{\odot} \mathrm{yr}^{-1}$; Xu et al. 2015) was detected in star formation tracers (Alonso-Herrero et al. 2001; Herrero-Illana et al. 2014; Pereira-Santaella et al. 2015; Saito et al. 2016a) and dense/warm gas tracers (Sliwa et al. 2014; Xu et al. 2015). The nucleus has no strong AGN (Imanishi \& Nakanishi 2013; Herrero-Illana et al. 2014; Pereira-Santaella et al. 2015) or heavily obscured AGN (Xu et al. 2015). The upper limit of the AGN luminosity suggests that it might be possible for driving the putative molecular gas outflow with the outflow rate of 40 $M_{\odot} \mathrm{yr}^{-1}$ as well as the starburst activities (García-Burillo et al. 2015). The detection of the molecular outflow coincides with the geometry of the ionized gas outflow with no redshifted component due to the extinction by the large column of dust in the inclined disk (Bellocchi et al. 2012).

Radiative transfer modeling with RADEX suggest that the starburst ring will have a single-phase $(\sim 42 \mathrm{~K})$ or two-phase ( $\sim 19$ and $>70 \mathrm{~K})$ molecular gas ISM, while the outer disk has a single cold gas ISM $(\sim 22 \mathrm{~K})$. The single-phase model is consistent with the FUV radiation field estimated by PDR models. However, star-forming activities are not enough to power the warm gas component in the starburst ring if the twophase ISM is valid. Alternatively, we suggest the mechanical heating from the supernovae and stellar winds as a heating source of the warm gas component.

\section{Conclusion}

In this paper, we present a detailed study of the molecular gas ISM in the luminous merger remnant NGC 1614 through high-resolution, high-sensitivity, and $u v$-matched ALMA observations of the $\mathrm{CO}(1-0), \mathrm{CO}(2-1), \mathrm{CO}(3-2)$, and $\mathrm{CO}(6-5)$ lines. The results are summarized as follows.

1. The CO (6-5) line shows a compact distribution that coincides with the starburst ring detected in $\mathrm{Pa} \alpha$ and radio-to-FIR continuum emission, while the other lower- $J$ CO lines are extended. This indicates that the $\mathrm{CO}(6-5)$ can be used as a better tracer of star formation as already suggested by $\mathrm{Xu}$ et al. (2015).

2. We find an "S"-shape non-circular motion along the nearIR north-south bar in the $\mathrm{CO}(1-0), \mathrm{CO}(2-1)$, and 

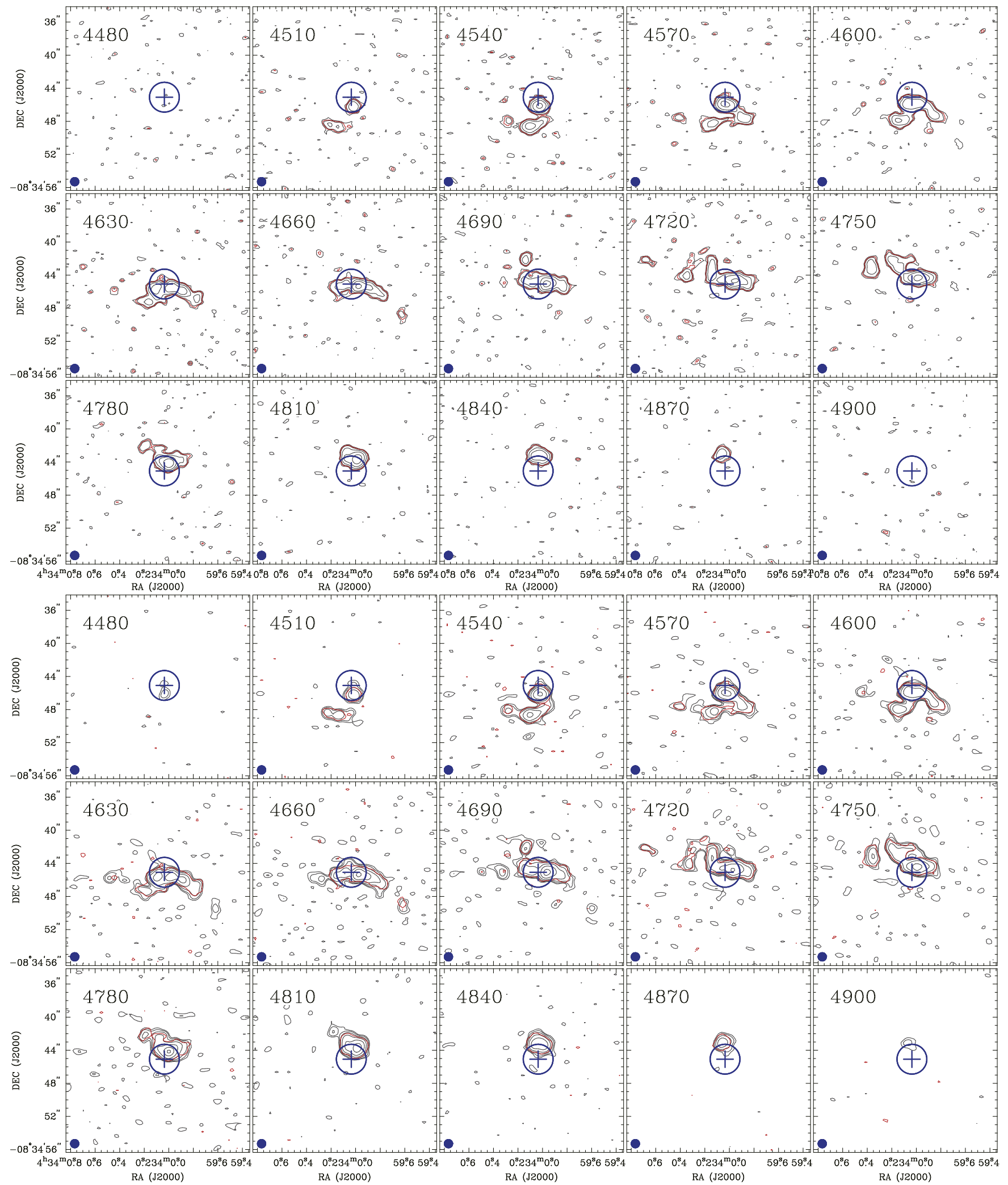

Figure 17. Channel maps (black contours) of CO (1-0) (top) and CO (2-1) (bottom) in NGC 1614. The $n$th contours are at $2^{n} \sigma(n=1,2,3 \ldots) . \sigma$ is the noise rms listed in Table 2. The red contours show the $4 \sigma$ level of the CO (1-0) as a comparison. The blue cross indicates the nucleus that is detected in Pa $\alpha$ and the radio continuum emission (Olsson et al. 2010; Herrero-Illana et al. 2014). The blue circle indicates the approximate outer edge of the nuclear ring (Herrero-Illana et al. 2014; Xu et al. 2015; Saito 2016a).

CO (3-2) velocity fields. Comparing with numerical simulations, this may be a bar-driven cold gas inflow connecting to the starburst ring.
3. Radiative transfer modeling with RADEX reveal that the molecular gas ISM in NGC 1614 can be described in a single-phase or two-phase model. The single-phase 


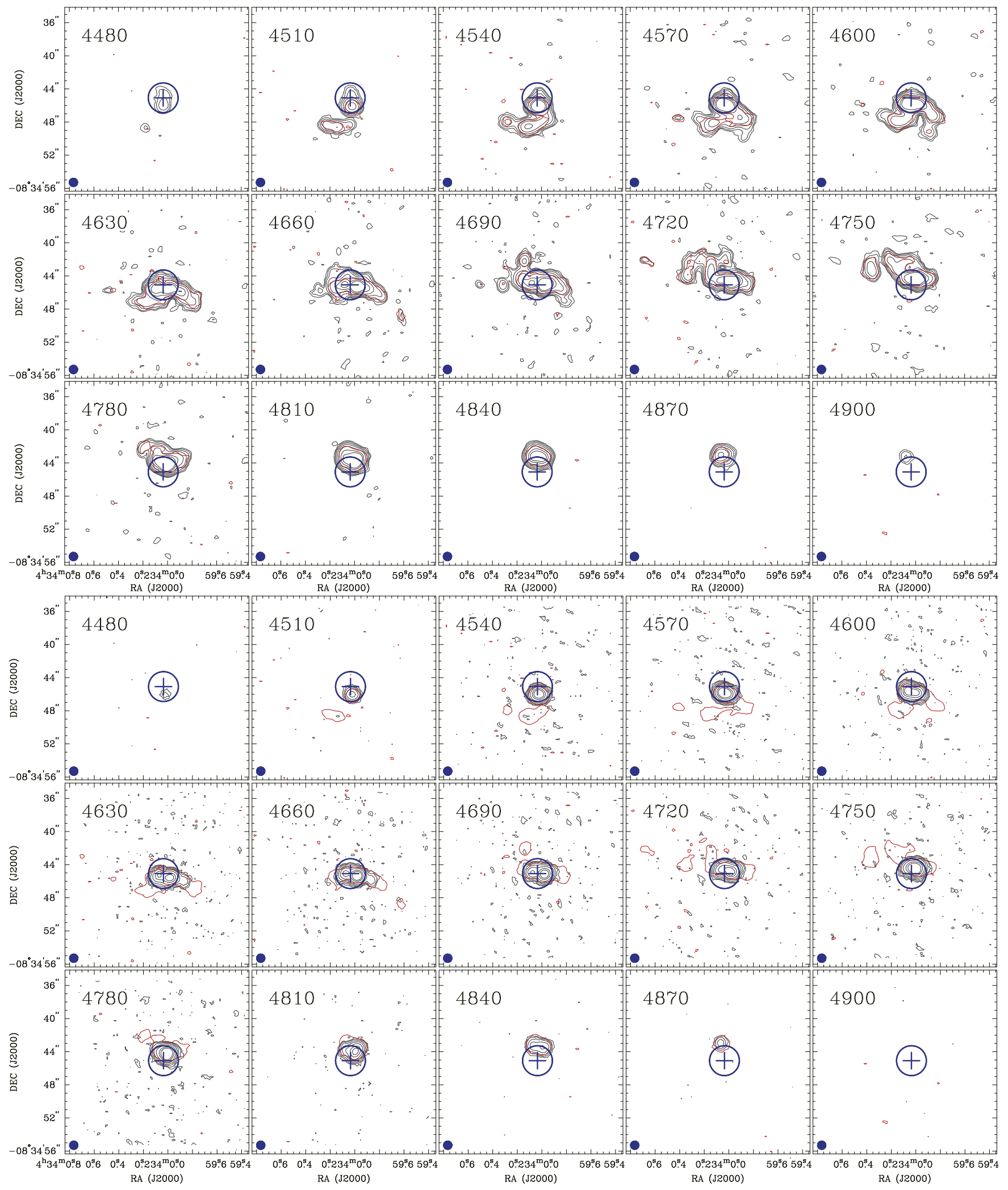

Figure 18. Same as Figure 17 but for CO (3-2) (top) and CO (6-5) (bottom).

model shows a radial gradient of the gas kinetic temperature from 70 to $20 \mathrm{~K}$ and the $\mathrm{H}_{2}$ column density from $10^{22.9}$ to $10^{22.0} \mathrm{~cm}^{-2}$ with peaks at the starburst ring. This single-phase model is applicable to explain the observed CO SLEDs, while there is a systematic underestimation of the $\mathrm{CO}(3-2)$ and $\mathrm{CO}(6-5)$ flux. The Narayanan \& Krumholz CO SLED model using the observed $\Sigma_{\text {SFR }}$ also shows the same trend. To account for the underestimation in both models, we verify the twophase model to the line ratios in the central $1 . ! 8$ radius. 


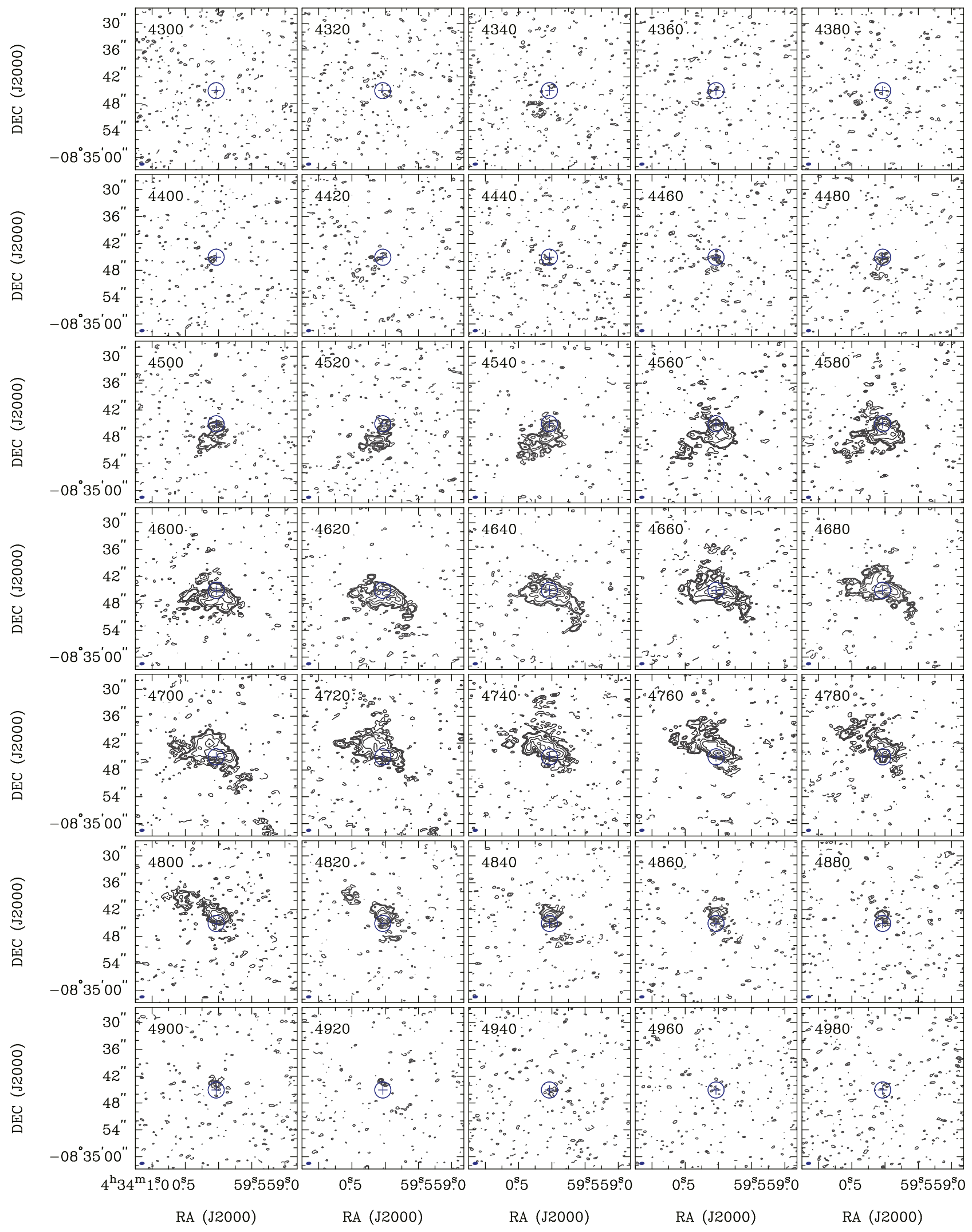

Figure 19. Same as Figure 17 for the ACA-combined CO (1-0). The contours are $1 \sigma \times\left(-2,2,3,4,8,16\right.$, and 32) $\mathrm{mJy}^{b^{2}} \mathrm{beam}^{-1}$.

4. The two-phase RADEX model for the starburst ring shows a good agreement with a cold gas component of $\sim 19 \mathrm{~K}$ and a warm gas component of $>70 \mathrm{~K}$, while there are number of assumptions at this stage. This is consistent with the two-phase dust ISM ( $\sim 35$ and $\sim 110 \mathrm{~K}$ ) revealed by high-resolution $(<0$ !" 5$)$ mid-IR and far-IR observations and two-phase modelings for other nearby U/LIRGs. A higher- $J$ CO observation $\left(J_{\text {upp }} \geqslant 7\right)$ is 
critical to characterize the molecular gas ISM in the starburst ring of NGC 1614.

5. Considering that the observed star-forming activities are not enough to power the warm gas component inferred from the two-phase RADEX, we suggest mechanical heating from supernovae and stellar winds as an alternative power source.

6. The summation between the cold molecular gas OFR and the SFR in the starburst ring is comparable to the cold gas IFR, showing evidence of the balance between the molecular gas input and output at the starburst ring. Assuming IFR, OFR, and SFR continue constantly, it takes dozens of $\mathrm{Myr}$ to exhaust all $\mathrm{H}_{2}$ mass in NGC 1614. This is consistent with the typical U/LIRG duration timescale suggested by numerical merger simulations.

We will present our high-resolution multi-transition observations, including $\mathrm{CO},{ }^{13} \mathrm{CO}, \mathrm{CN}$, CS, etc., toward NGC 1614 using Band 3 and Band 6 of ALMA in a forthcoming paper (M. Ando et al. 2017, in preparation).

The authors thanks an anonymous referee for comments that improved the contents of this paper. T.S. thanks N. Lu, E. W. Pellegrini, and E. Schinnerer for useful discussion. T.S. and the other authors thank the ALMA staff for their kind support and H. Nagai for the instruction of ALMA data reduction. T.S. and M.L. are financially supported by a Research Fellowship from the Japan Society for the Promotion of Science for Young Scientists. T.S. was supported by the ALMA Japan Research Grant of NAOJ Chile Observatory, NAOJ-ALMA-0114. D.I. was supported by the ALMA Japan Research Grant of NAOJ Chile Observatory, NAOJ-ALMA-0011, and JSPS KAKENHI grant No. 15H02074. This paper makes use of the following ALMA data: ADS/JAO.ALMA\#2011.0.00182.S, ADS/JAO. ALMA\#2011.0.00768.S, ADS/JAO.ALMA\#2013.1.00991.S, and ADS/JAO.ALMA\#2013.1.01172.S. ALMA is a partnership of ESO (representing its member states), NSF (USA) and NINS (Japan), together with NRC (Canada), NSC and ASIAA (Taiwan), and KASI (Republic of Korea), in cooperation with the Republic of Chile. The Joint ALMA Observatory is operated by ESO, AUI/NRAO and NAOJ. This research has made extensive use of the NASA/IPAC Extragalactic Database (NED) which is operated by the Jet Propulsion Laboratory, California Institute of Technology, under contract with the National Aeronautics and Space Administration.

\section{Appendix Channel Maps of the CO SLED of NGC 1614}

We show the channel map of the $u v$-matched CO (1-0), $\mathrm{CO}(2-1), \quad \mathrm{CO}(3-2)$, and $\mathrm{CO}(6-5)$ and ACA-combined CO (1-0) in Figures 17-19. These maps have the same angular resolution of 1 !' $0 \times 1$ !" 0 , the same velocity resolution of $30 \mathrm{~km} \mathrm{~s}^{-1}$, and the same MRS of $45 \mathrm{k} \lambda(\simeq 4$ ". 6 ), except for the ACA-combined CO (1-0) image. The lower- $J$ three transitions show almost same structures, although the $\mathrm{CO}(6-5)$ is only restricted within the central 2 ". 3 radius.

\section{References}

Aalto, S. 2013, in IAU Symp. 292, Molecular Gas, Dust, and Star Formation in Galaxies, ed. T. Wong \& J. Ott (Cambridge: Cambridge Univ. Press), 199 Albrecht, M., Krügel, E., \& Chini, R. 2007, A\&A, 462, 575
Alonso-Herrero, A., Engelbracht, C. W., Rieke, M. J., Rieke, G. H., \& Quillen, A. C. 2001, ApJ, 546, 952

Armus, L., Mazzarella, J. M., Evans, A. S., et al. 2009, PASP, 121, 559

Bayet, E., Williams, D. A., Hartquist, T. W., \& Viti, S. 2011, MNRAS, 414, 1583

Bellocchi, E., Arribas, S., \& Colina, L. 2012, A\&A, 542, A54

Bellocchi, E., Arribas, S., Colina, L., \& Miralles-Caballero, D. 2013, A\&A, 557, A59

Blake, G. A., Sutton, E. C., Masson, C. R., \& Phillips, T. G. 1987, ApJ, 315,621

Bolatto, A. D., Warren, S. R., Leroy, A. K., et al. 2013a, Natur, 499, 450

Bolatto, A. D., Wolfire, M., \& Leroy, A. K. 2013b, ARA\&A, 51, 207

Combes, F., García-Burillo, S., Casasola, V., et al. 2013, A\&A, 558, A124

Costagliola, F., Aalto, S., Rodriguez, M. I., et al. 2011, A\&A, 528, A30

Downes, D., \& Solomon, P. M. 1998, ApJ, 507, 615

Espada, D., Matsushita, S., Peck, A. B., et al. 2012, ApJL, 756, L10

Falstad, N., González-Alfonso, E., Aalto, S., et al. 2015, A\&A, 580, A52

García-Burillo, S., Combes, F., Usero, A., et al. 2014, A\&A, 567, A125

García-Burillo, S., Combes, F., Usero, A., et al. 2015, A\&A, 580, A35

Greve, T. R., Leonidaki, I., Xilouris, E. M., et al. 2014, ApJ, 794, 142

Hailey-Dunsheath, S., Sturm, E., Fischer, J., et al. 2012, ApJ, 755, 57

Herrero-Illana, R., Pérez-Torres, M. Á., Alonso-Herrero, A., et al. 2014, ApJ, 786,156

Hibbard, J. E., \& Yun, M. S. 1996, in Cold Gas at High Redshift, ed. M. N. Bremmer \& N. Malcol (Dordrecht: Kluwer), 47

Hollenbach, D. J., \& Tielens, A. G. G. M. 1999, RvMP, 71, 173

Iguchi, S., Morita, K.-I., Sugimoto, M., et al. 2009, PASJ, 61, 1

Imanishi, M., \& Nakanishi, K. 2013, AJ, 146, 47

Iono, D., Wilson, C. D., Takakuwa, S., et al. 2007, ApJ, 659, 283

Iono, D., Wilson, C. D., Yun, M. S., et al. 2009, ApJ, 695, 1537

Iono, D., Yun, M. S., \& Ho, P. T. P. 2005, ApJS, 158, 1

Iono, D., Yun, M. S., \& Mihos, J. C. 2004, ApJ, 616, 199

Kalnajs, A. J. 1978, in IAU Symp. 77, Structure and Properties of Nearby Galaxies, ed. E. M. Berkhuijsen \& R. Wielebinski (Dordrecht: Reidel), 113

Kamenetzky, J., Glenn, J., Rangwala, N., et al. 2012, ApJ, 753, 70

Kamenetzky, J., Rangwala, N., Glenn, J., Maloney, P. R., \& Conley, A. 2014, ApJ, 795, 174

Kamenetzky, J., Rangwala, N., Glenn, J., Maloney, P. R., \& Conley, A. 2016, ApJ, 829, 93

Kaneko, H., Kuno, N., Iono, D., et al. 2014, arXiv:1411.2660

Kaufman, M. J., Wolfire, M. G., \& Hollenbach, D. J. 2006, ApJ, 644, 283

König, S., Aalto, S., Muller, S., et al. 2016, A\&A, 594, A70

König, S., Aalto, S., Muller, S., Beswick, R. J., \& Gallagher, J. S. 2013, A\&A, 553, A72

Kotilainen, J. K., Reunanen, J., Laine, S., \& Ryder, S. D. 2001, A\&A, 366, 439

Leech, J., Isaak, K. G., Papadopoulos, P. P., Gao, Y., \& Davis, G. R. 2010, MNRAS, 406, 1364

Lin, L.-H., Wang, H.-H., Hsieh, P.-Y., et al. 2013, ApJ, 771, 8

Liu, D., Gao, Y., Isaak, K., et al. 2015, ApJL, 810, L14

Lu, N., Zhao, Y., Xu, C. K., et al. 2014, ApJL, 787, L23

Lundgren, A. 2013, ALMA Cycle 2 Technical Handbook Version 1.1, https:// almascience.nrao.edu/documents-and-tools/cycle-2/alma-technicalhandbook

Maloney, P. R. 1999, Ap\&SS, 266, 207

Mao, R.-Q., Schulz, A., Henkel, C., et al. 2010, ApJ, 724, 1336

Mashian, N., Sturm, E., Sternberg, A., et al. 2015, ApJ, 802, 81

Matsushita, S., Iono, D., Petitpas, G. R., et al. 2009, ApJ, 693, 56

McCray, R., \& Kafatos, M. 1987, ApJ, 317, 190

McMullin, J. P., Waters, B., Schiebel, D., Young, W., \& Golap, K. 2007, adass XVI, 376, 127

Meijerink, R., Kristensen, L. E., Weiß, A., et al. 2013, ApJL, 762, LL16

Meixner, M., \& Tielens, A. G. G. M. 1993, ApJ, 405, 216

Michiyama, T., Iono, D., Nakanishi, K., et al. 2016, PASJ, 68, 96

Narayanan, D., Groppi, C. E., Kulesa, C. A., \& Walker, C. K. 2005, ApJ, 630, 269

Narayanan, D., \& Krumholz, M. R. 2014, MNRAS, 442, 1411

Olsson, E., Aalto, S., Thomasson, M., \& Beswick, R. 2010, A\&A, 513, A11

Panuzzo, P., Rangwala, N., Rykala, A., et al. 2010, A\&A, 518, L37

Papadopoulos, P. P. 2010, ApJ, 720, 226

Papadopoulos, P. P., van der Werf, P. P., Xilouris, E. M., et al. 2012, MNRAS, 426, 2601

Papadopoulos, P. P., Zhang, Z.-Y., Xilouris, E. M., et al. 2014, ApJ, 788, 153 Pearson, C., Rigopoulou, D., Hurley, P., et al. 2016, arXiv:1610.06206 Pellegrini, E. W., Smith, J. D., Wolfire, M. G., et al. 2013, ApJL, 779, L19

Pereira-Santaella, M., Colina, L., Alonso-Herrero, A., et al. 2015, MNRAS, 454, 3679 
Pereira-Santaella, M., Spinoglio, L., Busquet, G., et al. 2013, ApJ, 768, 55

Pereira-Santaella, M., Spinoglio, L., van der Werf, P. P., \& Piqueras López, J. 2014, A\&A, 566, A49

Querejeta, M., Schinnerer, E., García-Burillo, S., et al. 2016, A\&A, 593, A118

Rangwala, N., Maloney, P. R., Glenn, J., et al. 2011, ApJ, 743, 94

Rangwala, N., Maloney, P. R., Wilson, C. D., et al. 2015, ApJ, 806, 17

Rigopoulou, D., Hurley, P. D., Swinyard, B. M., et al. 2013, MNRAS, 434, 2051

Rosenberg, M. J. F., van der Werf, P. P., Aalto, S., et al. 2015, ApJ, 801, 72

Rosenberg, M. J. F., van der Werf, P. P., \& Israel, F. P. 2012, A\&A, 540, A116

Rothberg, B., \& Joseph, R. D. 2004, AJ, 128, 2098

Saito, T., Iono, D., Xu, C. K., et al. 2016a, PASJ, 68, 20

Saito, T., Iono, D., Yun, M. S., et al. 2015, ApJ, 803, 60

Saitoh, T. R., Daisaka, H., Kokubo, E., et al. 2009, PASJ, 61, 481

Sanders, D. B., Scoville, N. Z., \& Soifer, B. T. 1991, ApJ, 370, 158

Sandstrom, K. M., Leroy, A. K., Walter, F., et al. 2013, ApJ, 777, 5

Schirm, M. R. P., Wilson, C. D., Parkin, T. J., et al. 2014, ApJ, 781, 101

Schöier, F. L., van der Tak, F. F. S., van Dishoeck, E. F., \& Black, J. H. 2005, A\&A, 432, 369

Sliwa, K., Wilson, C. D., Iono, D., Peck, A., \& Matsushita, S. 2014, ApJL, 796, L15

Sliwa, K., Wilson, C. D., Krips, M., et al. 2013, ApJ, 777, 126

Sliwa, K., Wilson, C. D., Petitpas, G. R., et al. 2012, ApJ, 753, 46
Solomon, P. M., \& Vanden Bout, P. A. 2005, ARA\&A, 43, 677

Spinoglio, L., Pereira-Santaella, M., Busquet, G., et al. 2012, ApJ, 758, 108

Tateuchi, K., Konishi, M., Motohara, K., et al. 2015, ApJS, 217, 1

Teyssier, R., Chapon, D., \& Bournaud, F. 2010, ApJL, 720, L149

Topal, S., Bayet, E., Bureau, M., Davis, T. A., \& Walsh, W. 2014, MNRAS, 437, 1434

Ueda, J., Iono, D., Yun, M. S., et al. 2014, ApJS, 214, 1

Väisänen, P., Rajpaul, V., Zijlstra, A. A., Reunanen, J., \& Kotilainen, J. 2012, MNRAS, 420, 2209

van der Tak, F. F. S., Black, J. H., Schöier, F. L., Jansen, D. J., \& van Dishoeck, E. F. 2007, A\&A, 468, 627

van der Werf, P. P., Isaak, K. G., Meijerink, R., et al. 2010, A\&A, 518, LL42

Viti, S., García-Burillo, S., Fuente, A., et al. 2014, A\&A, 570, A28

Wilson, C. D., Petitpas, G. R., Iono, D., et al. 2008, ApJS, 178, 189

Wilson, C. D., Rangwala, N., Glenn, J., et al. 2014, ApJL, 789, L36

Wilson, C. D., Warren, B. E., Israel, F. P., et al. 2012, MNRAS, 424, 3050

Wu, R., Madden, S. C., Galliano, F., et al. 2015, A\&A, 575, A88

Xu, C. K., Cao, C., Lu, N., et al. 2014, ApJ, 787, 48

Xu, C. K., Cao, C., Lu, N., et al. 2015, ApJ, 799, 11

Yao, L., Seaquist, E. R., Kuno, N., \& Dunne, L. 2003, ApJ, 588, 771

Zhao, Y., Lu, N., Xu, C. K., et al. 2016, ApJ, 820, 118

Zhu, M., Seaquist, E. R., \& Kuno, N. 2003, ApJ, 588, 243 